\title{
Google matrix of the citation network of Physical Review
}

\author{
Klaus M. Frahm, Young-Ho Eom, and Dima L. Shepelyansky \\ Laboratoire de Physique Théorique du CNRS, IRSAMC, Université de Toulouse, UPS, 31062 Toulouse, France
}

(Received 21 October 2013; published 28 May 2014)

\begin{abstract}
We study the statistical properties of spectrum and eigenstates of the Google matrix of the citation network of Physical Review for the period 1893-2009. The main fraction of complex eigenvalues with largest modulus is determined numerically by different methods based on high-precision computations with up to $p=16384$ binary digits that allow us to resolve hard numerical problems for small eigenvalues. The nearly nilpotent matrix structure allows us to obtain a semianalytical computation of eigenvalues. We find that the spectrum is characterized by the fractal Weyl law with a fractal dimension $d_{f} \approx 1$. It is found that the majority of eigenvectors are located in a localized phase. The statistical distribution of articles in the PageRank-CheiRank plane is established providing a better understanding of information flows on the network. The concept of ImpactRank is proposed to determine an influence domain of a given article. We also discuss the properties of random matrix models of Perron-Frobenius operators.
\end{abstract}

DOI: 10.1103/PhysRevE.89.052814

PACS number(s): $89.75 . \mathrm{Hc}, 89.20 . \mathrm{Hh}, 89.75 . \mathrm{Fb}$

\section{INTRODUCTION}

The development of the Internet has led to emergence of various types of complex directed networks created by modern society. The size of such networks has grown rapidly going beyond 10 billion in the last two decades for the World Wide Web (WWW). Thus the development of mathematical tools for the statistical analysis of such networks has become of primary importance. In 1998 Brin and Page proposed the analysis of WWW on the basis of the PageRank vector of the associated Google matrix constructed for a directed network [1]. The mathematical foundations of this analysis are based on Markov chains [2] and Perron-Frobenius operators [3]. The PageRank algorithm allows us to compute the ranking of network nodes and is known to be at the heart of modern search engines [4]. However, in many respects the statement of Brin and Page that "Despite the importance of large-scale search engines on the web, very little academic research has been done on them" [1] still remains valid at present. In our opinion, this is related to the fact that the Google matrix $G$ belongs to a new class of operators which had been rarely studied in physical systems. Indeed, the physical systems are usually described by Hermitian or unitary matrices for which random matrix theory [5] captures many universal properties. In contrast, the Perron-Frobenium operators and Google matrix have eigenvalues distributed in the complex plane belonging to another class of operators.

The Google matrix is constructed from the adjacency matrix $A_{i j}$, which has unit elements if there is a link pointing from node $j$ to node $i$ and zero otherwise. Then the matrix of Markov transitions is constructed by normalizing elements of each column to unity $\left(S_{i j}=A_{i j} / \sum_{i} A_{i j}, \sum_{j} S_{i j}=1\right)$ and replacing columns with only zero elements (dangling nodes) by $1 / N$, with $N$ being the matrix size. After that the Google matrix of the network takes the form $[1,4]$

$$
G_{i j}=\alpha S_{i j}+(1-\alpha) / N .
$$

The damping parameter $\alpha$ in the WWW context describes the probability $(1-\alpha)$ to jump to any node for a random surfer. For WWW the Google search engine uses $\alpha \approx 0.85$ [4]. The PageRank vector $P_{i}$ is the right eigenvector of $G$ at $\lambda=1$ $(\alpha<1)$. According to the Perron-Frobenius theorem [3], $P_{i}$ components are positive and represent the probability to find a random surfer on a given node $i$ (in the stationary limit) [4]. All nodes can be ordered in a decreasing order of probability $P\left(K_{i}\right)$ with highest probability at top values of PageRank index $K_{i}=1,2, \ldots$.

The distribution of eigenvalues of $G$ can be rather nontrivial with the appearance of the fractal Weyl law and other unusual properties (see, e.g., Refs. [6,7]). For example, a matrix $G$ with random positive matrix elements, normalized to unity in each column, has $N-1$ eigenvalues $\lambda$ concentrated in a small radius $|\lambda|<1 / \sqrt{3 N}$ and one eigenvalue $\lambda=1$ (see Sec. VII). Such a distribution is drastically different from the eigenvalue distributions found for directed networks with algebraic distribution of links [8] or those found numerically for other directed networks including universities' WWW [9,10], Linux Kernel and Twitter networks [11,12], and Wikipedia networks $[13,14]$. In fact, even the Albert-Barabási model of preferential attachment [15] still generates the complex spectrum of $\lambda$ with a large gap $(|\lambda|<1 / 2)$ [8] being very different from the gapless and strongly degenerate $G$ spectrum of British universities' WWW [10] and Wikipedia [13,14]. Thus it is useful to get a deeper understanding of the spectral properties of directed networks and to develop more advanced models of complex networks which have a spectrum similar to such networks as those of British universities and Wikipedia.

With the aim to understand the spectral properties of a Google matrix of directed networks we study here the citation network of Physical Review (CNPR) for the whole period up to 2009 [16]. This network has $N=463348$ nodes (articles) and $N_{\ell}=4691015$ links. Its network structure is very similar to the tree network since the citations are time ordered (with only a few exceptions of mutual citations of simultaneously published articles). As a result we succeed in developing powerful tools, which allowed us to obtain the spectrum of $G$ in semianalytical way. These results are compared with the spectrum obtained numerically with the help of the powerful Arnoldi method (see its description in Refs. [17,18]). Thus we are able to get a better understanding of the spectral properties of this network. Due to time ordering of article citations there are strong similarities between the CNPR and the network of integers studied recently in Refs. [19]. 
We note that the PageRank analysis of the CNPR had been performed in Refs. [20-23] showing its efficiency in determining the influential articles of Physical Review. The citation networks are rather generic (see, e.g., Ref. [24]), and hence the extension of PageRank analysis of such networks is an interesting and important task. Here we put the main accent on the spectrum and eigenstates properties of the Google matrix of the CNPR, but we also discuss the properties of two-dimensional (2D) ranking on the PageRank-CheiRank plane developed recently in Refs. [25-27]. We also analyze the properties of ImpactRank, which shows a domain of influence of a given article.

In addition to the whole CNPR we also consider the CNPR without Revview of Modern Physics articles, which has $N=$ 460 422, $N_{\ell}=4497707$. If in the whole CNPR we eliminate future citations (see description below), then this triangular CNPR has $N=463348, N_{\ell}=4684496$. Thus on average we have approximately 10 links per node. The network includes all articles of Physical Review from its foundation in 1893 till the end of 2009.

The paper is composed as follows: in Sec. II we present a detailed analysis of the Google matrix spectrum of CNPR, the fractal Weyl law is discussed in Sec. III, properties of eigenstates are discussed in Sec. IV, CheiRank versus PageRank distributions are considered in Sec. V, properties of impact propagation through the network are studied in Sec. VI, certain random matrix models of Google matrix are studied in Sec. VII, and discussion of the results is given in Section VIII.

\section{EIGENVALUE SPECTRUM}

The Google matrix of CNPR is constructed on the basis of Eq. (1) using citation links from one article to another (see also Refs. [21-23]). The matrix structure for different order representations of articles is shown in Fig. 1. In the top left panel all articles are ordered by time, which generates an almost perfect triangular structure corresponding to time ordering of citations. Still there are a few cases with joint citations of articles which appear almost at the same time. Also there are dangling nodes which generate transitions to all articles with elements $1 / N$ in $G$. This breaks the triangular structure, and as we will see later it is just the combination of these dangling node contributions with the other nonvanishing matrix elements [see also Eq. (4)] which will allow us to formulate a semianalytical theory to determine the eigenvalue spectrum.

The triangular matrix structure is also well visible in the middle left panel where articles are time ordered within each Physical Review journal. The left bottom panel shows the matrix elements for each Physical Review journal when inside each journal the articles are ordered by their PageRank index $K$. The right panels show the matrix elements of $G$ on different scales, when all articles are ordered by the PageRank index $K$. The top two right panels have a relatively small number of nonzero matrix elements showing that the top PageRank articles rarely quote other top PageRank articles.

The dependence of the number of no-zero links $N_{G}$, between nodes with PageRank index being less than $K$, on $K$ is shown in Fig. 2 (left panel). We see that compared to the other networks of universities, Wikipedia, and Twitter
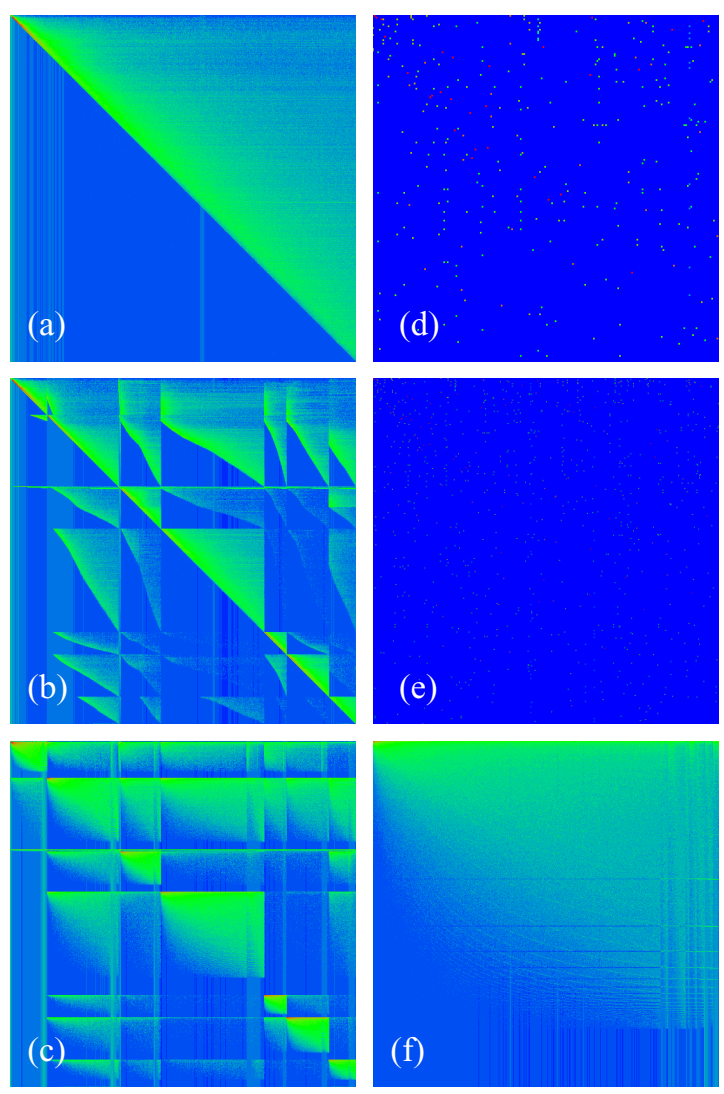

FIG. 1. (Color online) Different order representations of the Google matrix of the CNPR $(\alpha=1)$. The left panels show (a) the density of matrix elements $G_{t t^{\prime}}$ in the basis of the publication time index $t$ (and $t^{\prime}$ ); (b) the density of matrix elements in the basis of journal ordering according to Phys. Rev. Series I, Phys. Rev., Phys. Rev. Lett., Rev. Mod. Phys., Phys. Rev. A, B, C, D, E, Phys. Rev. STAB, and Phys. Rev. STPER with time ordering inside each journal; (c) the same as (b) but with PageRank index ordering inside each journal. Note that the journals Phys. Rev. Series I, Phys. Rev. STAB, and Phys. Rev. STPER are not clearly visible due to a small number of published papers. Also Rev. Mod. Phys. appears only as a thin line with 2-3 pixels (out of 500) due to a limited number of published papers. The panels (a), (b), (c), and (f) show the coarse-grained density of matrix elements done on $500 \times 500$ square cells for the entire network. In panels (d), (e), and (f) the matrix elements $G_{K K^{\prime}}$ are shown in the basis of PageRank index $K$ (and $K^{\prime}$ ) with the range $1 \leqslant K, K^{\prime} \leqslant 200(\mathrm{~d}) ; 1 \leqslant K, K^{\prime} \leqslant 400(\mathrm{e}) ; 1 \leqslant K, K^{\prime} \leqslant N$ (f). Color shows the amplitude (or density) of matrix elements $G$ changing from blue/black for zero value to red/gray at maximum value. The PageRank index $K$ is determined from the PageRank vector at $\alpha=0.85$.

studied in Ref. [13] we have for CNPR the lowest values of $N_{G} / K$ practically for all available $K$ values. This reflects weak links between top PageRank articles of CNPR in contrast with Twitter, which has a very high interconnection between top PageRank nodes. Since the matrix elements $G_{K K^{\prime}}$ are inversely proportional to the number of links, we have very strong average matrix elements for CNPR at top $K$ values (see Fig. 2, right panel).

In the following we present the results of numerical and analytical analysis of the spectrum of the CNPR matrix $G$. 

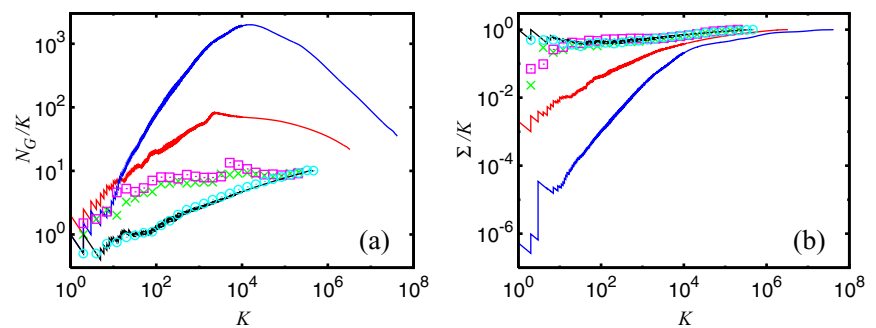

FIG. 2. (Color online) (a) Dependence of the linear density $N_{G} / K$ of nonzero elements of the adjacency matrix among top PageRank nodes on the PageRank index $K$ for the networks of Twitter (top blue/black curve), Wikipedia (second from top red/gray curve), Oxford University 2006 (magenta/gray boxes), and Cambridge University 2006 (green/gray crosses), with data taken from Ref. [12], and Physical Review all journals (cyan/gray circles) and Physical Review without Rep. Mod. Phys. (bottom black curve). (b) Dependence of the quantity $\Sigma / K$ on the PageRank index $K$ with $\Sigma=\sum_{K_{1}<K, K_{2}<K} G_{K_{1}, K_{2}}$ being the weight of the Google matrix elements inside the $K \times K$ square of top PageRank indexes. The curves correspond to the same networks as in (a): Physical Review without Rep. Mod. Phys. (top black curve), Physical Review all journals (cyan/gray circles), Oxford University 2006 (magenta/gray boxes), Cambridge University 2006 (green/gray crosses), Wikipedia (second bottom red/gray curve), and Twitter (bottom blue/black curve).

\section{A. Nearly nilpotent matrix structure}

The triangular structure of the CNPR Google matrix in the time index (see Fig. 1) has important consequences for the eigenvalue spectrum $\lambda$ defined by the equation for the eigenstates $\psi_{i}(j)$ :

$$
\sum_{j^{\prime}} G_{j j^{\prime}} \psi_{i}\left(j^{\prime}\right)=\lambda_{i} \psi_{i}(j)
$$

The spectrum of $G$ at $\alpha=1$, or the spectrum of $S$, obtained by the Arnoldi method [17,18] with the Arnoldi dimension $n_{A}=8000$, is shown in Fig. 3. For comparison we also show the case of a reduced CNPR without Review of Modern Physics. We see that the spectrum of the reduced case is rather similar to the spectrum of the full CNPR.

The nodes can be decomposed in invariant subspace nodes and core space nodes, and the matrix $S$ can be written in the block structure [10]:

$$
S=\left(\begin{array}{cc}
S_{s s} & S_{s c} \\
0 & S_{c c}
\end{array}\right)
$$

where $S_{s s}$ contains the links from subspace nodes to other subspace nodes, $S_{c c}$ the links from core space nodes to core space nodes, and $S_{s c}$ some coupling links from the core space to the invariant subspaces. The subspace-subspace block $S_{s s}$ is actually composed of (potentially) many diagonal blocks for each of the invariant subspaces. Each of these blocks corresponds to a column sum-normalized matrix of the same type as $G$ and has therefore at least one unit eigenvalue, thus explaining a possible high degeneracy of the eigenvalue $\lambda=1$ of $S$. This structure is discussed in detail in Ref. [10]. The university networks discussed in Ref. [10] had a considerable number of subspace nodes (about 20\%) with a high degeneracy
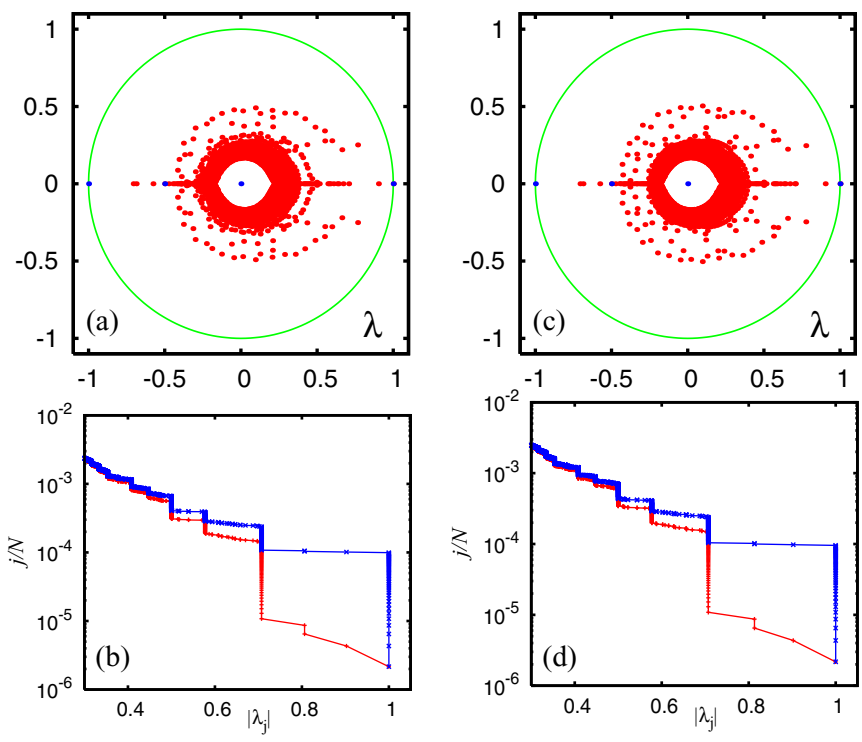

FIG. 3. (Color online) Spectrum of $S$ for CNPR (reduced CNPR without Rev. Mod. Phys.) shown on left panels (right panels). (a, c) Subspace eigenvalues (blue/black dots) and core space eigenvalues (red/gray dots) in the $\lambda$ plane (green/gray curve shows unit circle); there are 27 (26) invariant subspaces, with maximal dimension 6 (6), and the sum of all subspace dimensions is $N_{s}=71$ (75). The core space eigenvalues are obtained from the Arnoldi method applied to the core space subblock $S_{c c}$ of $S$ with Arnoldi dimension $n_{A}=8000$ as explained in Ref. [10] and using standard double-precision arithmetic. (b, d) Fraction $j / N$ of eigenvalues, shown in a logarithmic scale, with $|\lambda|>\left|\lambda_{j}\right|$ for the core space eigenvalues (red/gray bottom curve) and all eigenvalues (blue/black top curve) from raw data of top panels. The number of eigenvalues with $\left|\lambda_{j}\right|=1$ is 45 (43) of which 27 (26) are at $\lambda_{j}=1$; this number is identical to the number of invariant subspaces which have each one unit eigenvalue.

$\sim 10^{3}$ of the leading unit eigenvalue. However, for the CNPR the number of subspace nodes and unit eigenvalues is quite small (see the figure caption of Fig. 3 for detailed values).

A network with a similar triangular structure, constructed from factor decompositions of integer numbers, was previously studied in Ref. [19]. There it was analytically shown that the corresponding matrix $S$ has only a small number of nonvanishing eigenvalues and that the numerical diagonalization methods, including the Arnoldi method, are facing subtle difficulties of numerical stability due to large Jordan blocks associated to the highly degenerate zero eigenvalue. The numerical diagonalization of these Jordan blocks is highly sensitive to numerical round-off errors. For example, a perturbed Jordan block of dimension $D$ associated to the eigenvalue zero and with a perturbation $\varepsilon$ in the opposite corner has eigenvalues on a complex circle of radius $\varepsilon^{1 / D}$ [19], which may became quite large for sufficient large $D$ even for $\varepsilon \sim 10^{-15}$. Therefore in the presence of many such Jordan blocks the numerical diagonalization methods create rather big "artificial clouds" of incorrect eigenvalues.

In the examples studied in Ref. [19] these clouds extended up to eigenvalues $|\lambda| \approx 0.01$. The spectrum for the Physical Review network shown in Fig. 3 shows also a sudden 
increase of the density of eigenvalues below $|\lambda| \approx 0.3-0.4$, and one needs to be concerned if these eigenvalues are numerically correct or only an artifact of the same type of numerical instability. Actually there is a quite simple way to verify that they are not reliable due to problems in the numerical evaluation. For this we apply to the network or the numerical algorithm (in the computer program) certain transformations or modifications which are mathematically neutral or equivalent, e.g., a permutation of the index numbers of the network nodes but keeping the same network-link structure, or simply changing the evaluation order in the sums used for the scalar products between vectors (in the Gram-Schmidt orthogonalization for the Arnoldi method). All these modifications should in theory not modify the results (assuming that all computations could be done with infinite precision), but in numerical computations on a computer with finite precision they modify the round-off errors. It turns out indeed that the modifications of the initially small round-off errors induce very strong, completely random modifications, for all eigenvalues below $|\lambda| \approx 0.3-0.4$, clearly indicating that the latter are numerically not accurate. Apparently the problematic numerical eigenvalue errors due to large Jordan blocks $\sim \varepsilon^{1 / D}$ with $D \sim 10^{2}$ is quite stronger in the Physical Review citation network than in the previously studied integer network [19].

The theory of Ref. [19] is based on the exact triangular structure of the matrix $S_{0}$, which appears in the representation of $S=S_{0}+e d^{T} / N$ [see also Eq. (4)]. In fact, the matrix $S_{0}$ is obtained from the adjacency matrix by normalizing the sum of the elements in nonvanishing columns to unity and simply keeping at zero vanishing columns. For the network of integers [19] this matrix is nilpotent with $S_{0}^{l}=0$ for a certain modest value of $l$ being much smaller than the network size $l \ll N$. The nilpotency is very relevant in the paper for two reasons: first, it is responsible for the numerical problems to compute the eigenvalues by standard methods (see the next point), and, second, it is also partly the solution by allowing a semianalytical approach to determine the eigenvalues in a different way.

For CNPR the matrix $S_{0}$ is not exactly nilpotent despite the overall triangular matrix structure visible in Fig. 1. Even though most of the nonvanishing matrix elements $\left(S_{0}\right)_{t t^{\prime}}$ (whose total number is equal to the number of links $N_{\ell}=$ $4691015)$ are in the upper triangle $t<t^{\prime}$ there are a few nonvanishing elements in the lower triangle $t>t^{\prime}$ (whose number is 12126 corresponding to $0.26 \%$ of the total number of links [28]). The reason is that in most cases papers cite other papers published earlier but in certain situations for papers with a close publication date the citation order does not always coincide with the publication order. In some cases two papers even mutually cite each other. In the following we will call these cases "future citations." The rare nonvanishing matrix elements due to future citations are not visible in the coarse-grained matrix representation of Fig. 1, but they are responsible for the fact that $S_{0}$ of CMPR is not nilpotent and that there are also a few invariant subspaces. On a purely triangular network one can easily show the absence of invariant subspaces (smaller than the full network size) when taking into account the extra columns due to the dangling nodes.
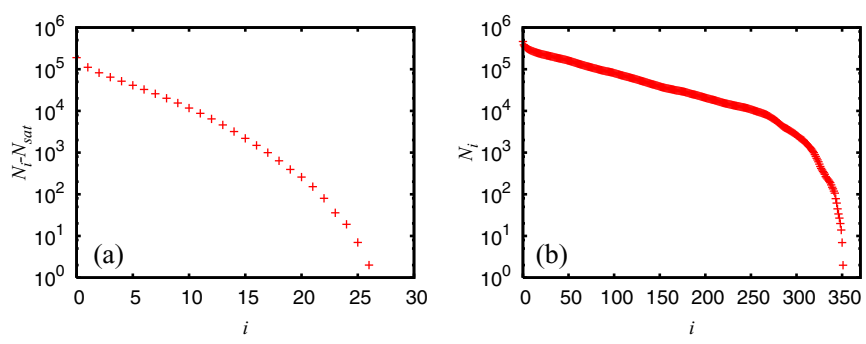

FIG. 4. (Color online) Number of occupied nodes $N_{i}$ (i.e., positive elements) in the vector $S_{0}^{i} e$ versus iteration number $i$ (red/gray crosses) for the CNPR (a) and the triangular CNPR (b). In both cases the initial value is the network size $N_{0}=N=463348$. For the CNPR $N_{i}$ saturates at $N_{i}=N_{\text {sat }}=273490 \approx 0.590 N$ for $i \geqslant 27$ while for the triangular CNPR $N_{i}$ saturates at $N_{i}=0$ for $i \geqslant 352$ confirming the nilpotent structure of $S_{0}$. In (a) the quantity $N_{i}-N_{\text {sat }}$ is shown in order to increase visibility in the logarithmic scale.

However, despite the effect of the future citations the matrix $S_{0}$ is still partly nilpotent. This can be seen by multiplying a uniform initial vector $e$ (with all components being 1) by the matrix $S_{0}$ and counting after each iteration the number $N_{i}$ of nonvanishing entries [29] in the resulting vector $S_{0}^{i} e$. For a nilpotent matrix $S_{0}$ with $S_{0}^{l}=0$ the number $N_{i}$ becomes obviously zero for $i \geqslant l$. On the other hand, since the components of $e$ and the nonvanishing matrix elements of $S_{0}$ are positive, one can easily verify that the condition $S_{0}^{l} e=0$ for some value $l$ also implies $S_{0}^{l} \psi=0$ for an arbitrary initial (even complex) vector $\psi$, which shows that $S_{0}$ must be nilpotent with $S_{0}^{l}=0$.

In Fig. 4 we see that for the CNPR the value of $N_{i}$ saturates at a value $N_{\text {sat }}=273490$ for $i \geqslant 27$, which is $59 \%$ of the total number of nodes $N=463348$ in the network. On one hand the (small) number of future citations ensures that the saturation value of $N_{i}$ is not zero, but on the other hand it is smaller than the total number of nodes by a macroscopic factor. Mathematically the first iteration $e \rightarrow S_{0} e$ removes the nodes corresponding to empty (vanishing) lines of the matrix $S_{0}$, and the next iterations remove the nodes whose lines in $S_{0}$ have become empty after having removed from the network the nonoccupied nodes due to previous iterations. For each node removed during this iteration process one can construct a vector belonging to the Jordan subspace of $S_{0}$ associated to the eigenvalue 0 . In the following we call this subspace generalized kernel. It contains all eigenvectors of $S_{0}^{j}$ associated to the eigenvalue 0 where the integer $j$ is the size of the largest 0-eigenvalue Jordan block. Obviously the dimension of this generalized kernel of $S_{0}$ is larger or equal than $N-N_{\text {sat }}=$ 189857 , but we will see later that its actual dimension is even larger and quite close to $N$. We will argue below that most (but not all) of the vectors in the generalized kernel of $S_{0}$ also belong to the generalized kernel of $S$, which differs from $S_{0}$ by the extra contributions due to the dangling nodes. The high dimension of the generalized kernel containing many large 0 -eigenvalue Jordan subspaces explains very clearly the numerical problem due to which the eigenvalues obtained by the double-precision Arnoldi method are not reliable for $|\lambda|<$ $0.3-0.4$. 


\section{B. Spectrum for the triangular CNPR}

In order to extend the theory for the triangular matrices developed in Ref. [19] we consider the triangular CNPR obtained by removing all future citation links $t^{\prime} \rightarrow t$ with $t \geqslant t^{\prime}$ from the original CNPR. The resulting matrix $S_{0}$ of this reduced network is now indeed nilpotent with $S_{0}^{l-1} \neq 0$, $S_{0}^{l}=0$, and $l=352$, which is much smaller than the network size. This is clearly seen from Fig. 4, showing that $N_{i}$, calculated from the triangular CNPR, indeed saturates at $N_{i}=0$ for $i \geqslant 352$. According to the arguments of Ref. [19], and additional demonstrations given below, there are at most only $l=352$ nonzero eigenvalues of the Google matrix at $\alpha=1$. This matrix has the form

$$
S=S_{0}+(1 / N) e d^{T}
$$

where $d$ and $e$ are two vectors with $e(n)=1$ for all nodes $n=$ $1, \ldots, N$ and $d(n)=1$ for dangling nodes $n$ (corresponding to vanishing columns in $S_{0}$ ) and $d(n)=0$ for the other nodes. In the following we call $d$ the dangling vector. The extra contribution $e d^{T} / N$ just replaces the empty columns (of $S_{0}$ ) with $1 / N$ entries at each element and $d^{T}$ is the line vector obtained as the transpose of the column vector $d$. In Appendix A we extend the approach of Ref. [19] showing analytically that the matrix $S$ has exactly $l=352 \ll N$ nonvanishing eigenvalues, which are given as the zeros of the reduced polynomial given in Eq. (A4), and that it is possible to define a closed representation space for the matrix $S$ of dimension $l$ leading to an $l \times l$ representation matrix $\bar{S}$ given by Eq. (A8) whose eigenvalues are exactly the zeros of the reduced polynomial.

In the left panel of Fig. 5 we compare the core space spectrum of $S$ for CNPR and triangular CNPR (data are obtained by the Arnoldi method with $n_{A}=4000$ and standard double precision). We see that the largest complex eigenvalues are rather close for both cases, but in the full network we have many eigenvalues on the real axis (with $\lambda<-0.3$ or $\lambda>0.4$ ), which are absent for the triangular CNPR. Furthermore, both
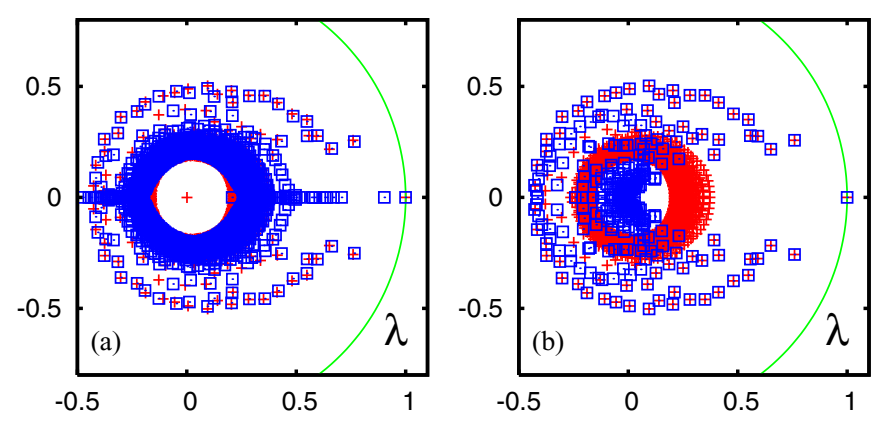

FIG. 5. (Color online) (a) Comparison of the core space eigenvalue spectrum of $S$ for CNPR (blue/black squares) and triangular CNPR (red/gray crosses). Both spectra are calculated by the Arnoldi method with $n_{A}=4000$ and standard double precision. (b) Comparison of the numerically determined nonvanishing 352 eigenvalues obtained from the representation matrix (A8) (blue/black squares) with the spectrum of triangular CNPR (red/gray crosses) already shown in the left panel. Numerics is done with standard double precision. cases suffer from the same problem of numerical instability due to large Jordan blocks.

In the right panel of Fig. 5 we compare the numerical double-precision spectra of the representation matrix $\bar{S}$ with the results of the Arnoldi method with double precision and the uniform initial vector $e$ as start vector for the Arnoldi iterations (applied to the triangular CNPR). In Appendix B we explain that the Arnoldi method with this initial vector should in theory (in absence of rounding errors) also exactly provide the $l$ eigenvalues of $\bar{S}$ since by construction it explores the same $l$-dimensional $S$-invariant representation space that was used for the construction of $\bar{S}$ (in Appendix A). The fact that both spectra of the right panel of Fig. 5 differ is therefore a clear effect of numerical errors, and actually both cases suffer from different numerical problems (see Appendix B for details). A different, and in principle highly efficient, computational method is to calculate the spectrum of the triangular CNPR by determining numerically the $l$ zeros of the reduced polynomial (A4), but according to the further discussion in Appendix B there are also numerical problems for this. Actually this method requires the help the GNU Multiple Precision Arithmetic Library (GMP library) [30] using 256 binary digits. Also the Arnoldi method can be improved by GMP library (see Appendix B for details) even though this is quite expensive in computational time and memory usage but still feasible (using up to 1280 binary digits). Below we will also present results (for the spectrum of the full CNPR) based on a new method using the GMP library with up to 16384 binary digits.

In Fig. 6 we compare the exact spectrum of the triangular CNPR obtained by the high-precision determination of the zeros of the reduced polynomial (using 256 binary digits) with the spectra of the Arnold method for 52 binary digits (corresponding to the mantissa of double-precision numbers), 256, 512, and 1280 binary digits. Here we use for the Arnoldi method a uniform initial vector and the Arnold dimension $n_{A}=l=352$. In this case, as explained in Appendix B, in theory the Arnoldi method should provide the exact $l=352$ nonvanishing eigenvalues (in the absence of round-off errors).

However, with the precision of 52 bits we have a considerable number of eigenvalues on a circle of radius $\approx 0.3$ centered at 0.05 , indicating a strong influence of round-off errors due to the Jordan blocks. Increasing the precision to 256 (or 512) binary digits implies that the number of correct eigenvalue increases and the radius of this circle decreases to 0.13 (or 0.1 ), and in particular it does not extend to all angles. We have to increase the precision of the Arnoldi method to 1280 binary digits to have a perfect numerical confirmation that the Arnoldi method explores the exact invariant subspace of dimension $l=352$ and generated by the vectors $v_{j}$ (see Appendix A). In this case the eigenvalues obtained from the Arnoldi method and the high-precision zeros of the reduced polynomial coincide with an error below $10^{-14}$ and in particular the Arnoldi method provide a nearly vanishing coupling matrix element at the last iteration, confirming that there is indeed an exact decoupling of the Arnoldi matrix and an invariant closed subspace of dimension 352.

The results shown in Fig. 6 clearly confirm the above theory and the scenario of the strong influence of Jordan blocks on the round-off errors. In particular, we find that in order to increase 

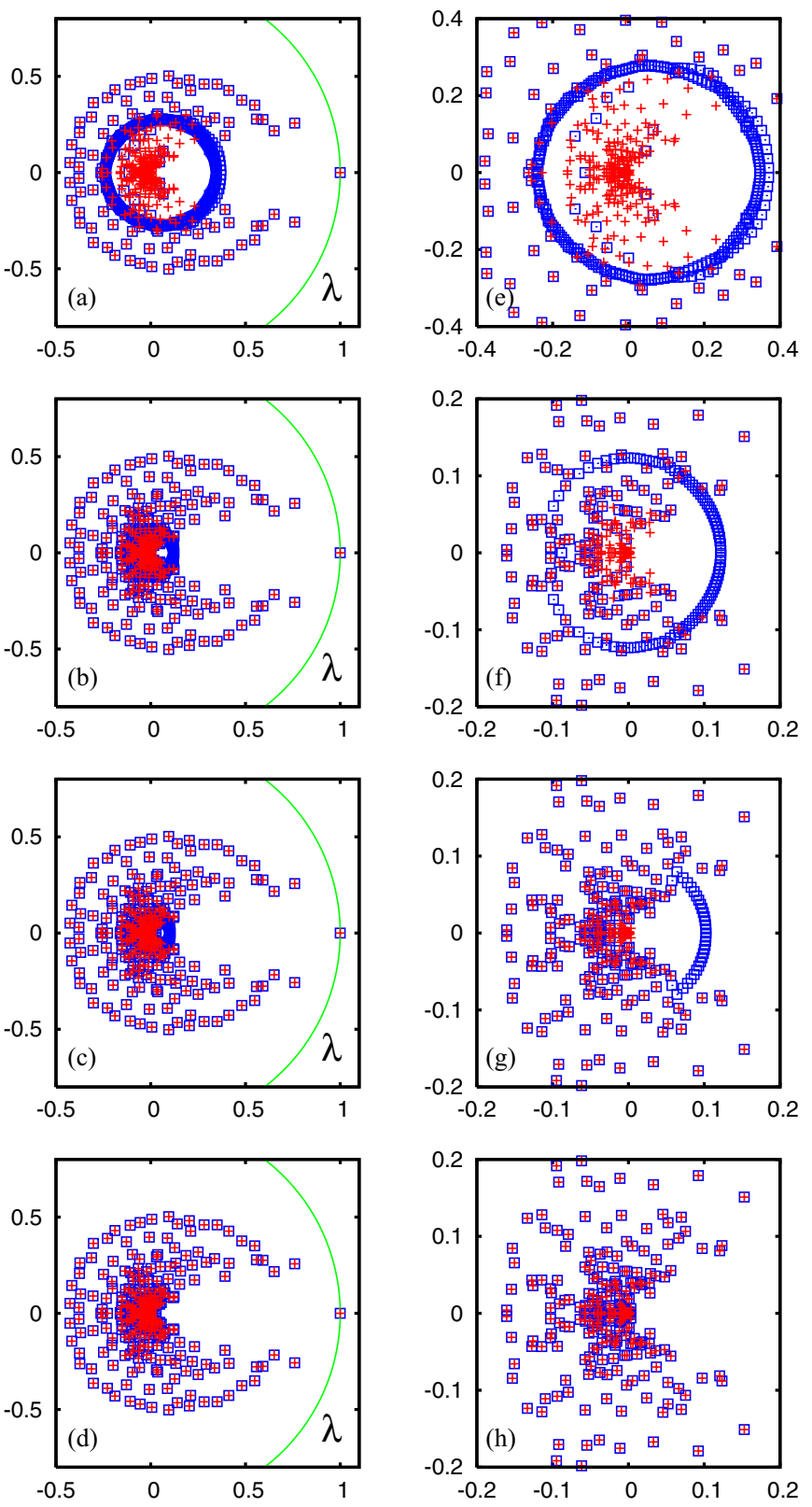

FIG. 6. (Color online) Comparison of the numerically accurate 352 nonvanishing eigenvalues of $S$ matrix of triangular CNPR, determined by the Newton-Maehly method applied to the reduced polynomial (A4) with a high-precision calculation of 256 binary digits (red/gray crosses, all panels), with eigenvalues obtained by the Arnoldi method at different numerical precisions (for the determination of the Arnoldi matrix) for triangular CNPR and Arnoldi dimension $n_{A}=352$ (blue/black squares, all panels). The first row corresponds to the numerical precision of 52 binary digits for standard double-precision arithmetic. The second (third, fourth) row corresponds to the precision of $256(512,1280)$ binary digits. All high-precision calculations are done with the library GMP [30]. The panels in the left column show the complete spectra and the panels in the right columns show the spectra in a zoomed range: $-0.4 \leqslant$ $\operatorname{Re}(\lambda), \operatorname{Im}(\lambda) \leqslant<0.4$ for the first row or $-0.2 \leqslant \operatorname{Re}(\lambda), \operatorname{Im}(\lambda) \leqslant 0.2$ for the second, third, and fourth rows.

the numerical precision it is only necessary to implement the first step of the method, the Arnoldi iteration, using high-precision numbers while the numerical diagonalization of the Arnoldi representation matrix can still be done using standard double-precision arithmetic. We also observe that even for the case with lowest precision of 52 binary digits the eigenvalues obtained by the Arnoldi method are numerically accurate provided that there are well outside the circle (or cloud) of numerically incorrect eigenvalues.

\section{High-precision spectrum of the whole CNPR}

Based on the observation that a high-precision implementation of the Arnoldi method is useful for the triangular CNPR, we now apply the high-precision Arnoldi method with 256, 512 , and 756 binary digits and $n_{A}=2000$ to the original CNPR. The results for the core space eigenvalues are shown in Fig. 7, where we compare the spectrum of the highest precision of 756 binary digits with lower-precision spectra of 52, 256, and 512 binary digits. As in Fig. 6 for the triangular CNPR, for CNPR we also observe that the radius and angular extension of the cloud or circle of incorrect Jordan block eigenvalues decrease with increasing precision. Despite the lower number of $n_{A}=2000$ as compared to $n_{A}=8000$ of Fig. 3 the number of accurate eigenvalues with 756-bit precision is certainly considerably higher.

The higher-precision Arnoldi method certainly improves the quality of the smaller eigenvalues, e.g., for $|\lambda|<0.3-0.4$,
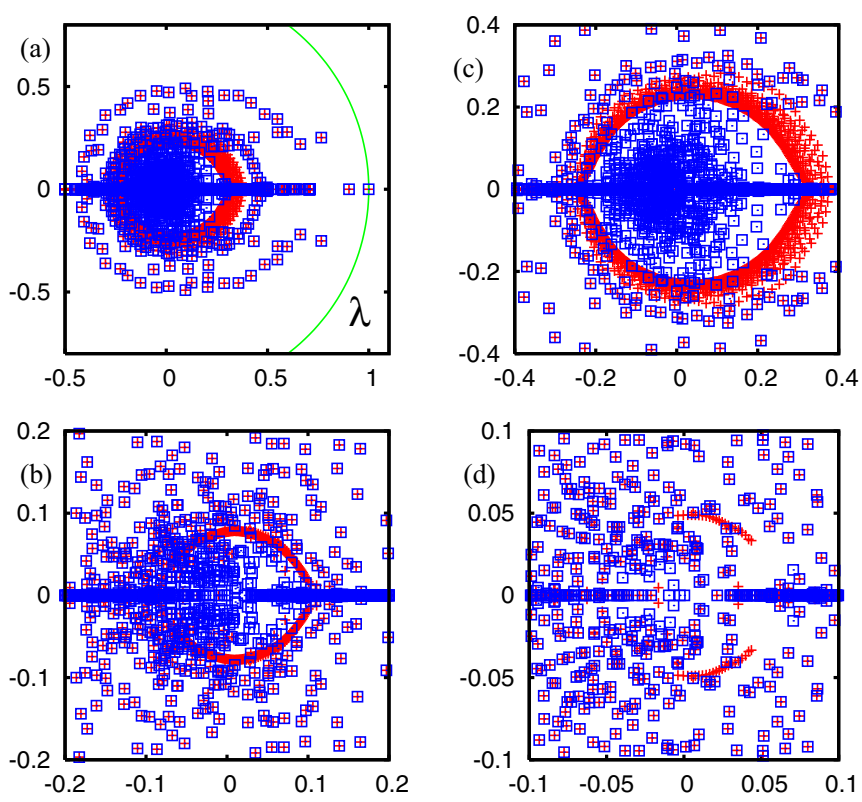

FIG. 7. (Color online) Comparison of the core space eigenvalue spectrum of $S$ of CNPR, obtained by the high-precision Arnoldi method using 768 binary digits (blue/black squares, all panels), with lower-precision data of the Arnoldi method (red/gray crosses). In both top panels the red/gray crosses correspond to double precision with 52 binary digits [extended range in (a) and zoomed range in (c)]. In the bottom (b) [or (d))] panel red/gray crosses correspond to the numerical precision of 256 (or 512) binary digits. In these two cases only a zoomed range is shown. The eigenvalues outside the zoomed ranges coincide for both data sets up to graphical precision. In all cases the Arnoldi dimension is $n_{A}=2000$. High-precision calculations are done with the library GMP [30]. 

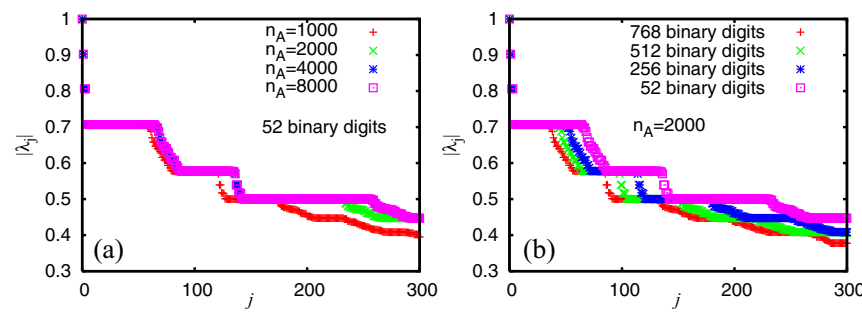

FIG. 8. (Color online) Modulus $\left|\lambda_{j}\right|$ of the core space eigenvalues of $S$ of CNPR, obtained by the Arnoldi method, shown versus level number $j$. (a) Data for standard double precision with 52 binary digits with different Arnoldi dimensions $1000 \leqslant n_{A} \leqslant 8000$. (b) Data for Arnoldi dimension $n_{A}=2000$ with different numerical precisions between 52 and 768 binary digits.

but it also implies a strange shortcoming as far as the degeneracies of certain particular eigenvalues are concerned. This can be seen in Fig. 8, which shows the core space eigenvalues $\left|\lambda_{j}\right|$ versus the level number $j$ for various values of the Arnoldi dimension and the precision. In these curves we observe flat plateaux at certain values $\left|\lambda_{j}\right|=1 / \sqrt{n}$ with $n=2,3,4,5, \ldots$ corresponding to degenerate eigenvalues which turn out to be real but with positive or negative values: $\lambda_{j}= \pm 1 / \sqrt{n}$. For fixed standard double-precision arithmetic with 52 binary digits the degeneracies increase with increasing Arnoldi dimension and seem to saturate for $n_{A} \geqslant 4000$. However, at the given value of $n_{A}=2000$ the degeneracies decrease with increasing precision of the Arnoldi method. Apparently the higher-precision Arnoldi method is less able to determine the correct degeneracy of a degenerate eigenvalue.

This point can be understood as follows. In theory, assuming perfect precision, the simple version of the Arnoldi method used here (in contrast to more complicated block Arnoldi methods) can determine only one eigenvector for a degenerate eigenvalue. The reason is that for a degenerate eigenvalue we have a particular linear combination of the eigenvectors for this eigenvalue which contribute in any initial vector (in other words "one particular" eigenvector for this eigenvalue), and during the Arnoldi iteration this particular eigenvector will be perfectly conserved and the generated Krylov space will contain only this and no other eigenvector for this eigenvalue. However, due to round-off errors we obtain at each step new random contributions from other eigenvectors of the same eigenvalue, and it is due only to these round-off errors that we can see the flat plateaux in Fig. 8. Obviously, increasing the precision reduces this round-off error effect, and the flat plateaux are indeed considerably smaller for higher precisions.

The question arises about the origin of the degenerate eigenvalues in the core space spectrum. In other examples, such as the WWW for certain university networks [10], the degeneracies, especially of the leading eigenvalue 1 , could be treated by separating and diagonalizing the exact subspaces, and the remaining core space spectrum contained much less or nearly no degenerate eigenvalues. However, here for the CNPR we have "only" 27 subspaces with maximal dimension of 6 containing 71 nodes in total. The eigenvalues due to these subspaces are $1,-1,-0.5$, and 0 with degeneracies 27, 18, 4, and 22 (see blue dots in the upper panels of Fig. 3). These exact subspaces exist due only to the modest number of future citation links. Even when we take care that in all cases the Arnoldi method is applied to the core space without these 71 subspace nodes, there still remain many degenerate eigenvalues in the core space spectrum.

In Appendix $\mathrm{C}$ we explain how the degenerated core space eigenvalues of $S$ can be obtained as degenerate subspace eigenvalues of $S_{0}$ (i.e., neglecting the dangling node contributions when determining the invariant subspaces). To be precise it turns out that the core space eigenvalues of $S$ are decomposed in two groups: the first group is related to degenerate subspace eigenvalues of $S_{0}$ and can be determined by a scheme described in Appendix $\mathrm{C}$, and the second group of eigenvalues is given as zeros of a certain rational function ((D1), which can be evaluated by the series (D3) which converges only for $|\lambda|>\rho_{1}$ with $\rho_{1} \approx 0.902$. To determine the zeros of the rational function, outside the range of convergence, one can employ an argument of analytical continuation using a new method, called "rational interpolation method" described in detail in Appendix D. Without going into much detail here, we mention that the main idea of this method is to evaluate this rational function at many support points on the complex unit circle where the series (D3) converges well and then to use these values to interpolate the rational function (D1) by a simpler rational function for which the zeros can be determined numerically well even if they are inside the unit circle (where the initial series does not converge). For this scheme it is also very important to use high-precision computations. Typically for a given precision of $p$ binary digits one may chose a certain number $n_{R}$ of eigenvalues to be determined choosing the appropriate number of support points (either $2 n_{R}+1$ or $2 n_{R}+2$ depending on the variant of the method; see also Appendix D). Provided that $n_{R}$ is not neither too small nor too large (depending on the value of $p$ ) one obtains very reliable core space eigenvalues of $S$ of the second group.

For example, as can be seen in Fig. 9, for $p=1024$ we obtain $n_{R}=300$ eigenvalues for which the big majority coincides numerically (error $\sim 10^{-14}$ ) with the eigenvalues obtained from the high-precision Arnoldi method for 768 binary digits, and furthermore both variants of the rational interpolation method provide identical spectra.

However, for $n_{R}=340$ some of the zeros do not coincide with eigenvalues of $S$, and most of these deviating zeros lie close to the unit circle. We can even somehow distinguish between "good" zeros (associated to eigenvalues of $S$ ) being identical for both variants of the method and "bad" artificial zeros, which are completely different for both variants (see Fig. 9). We note that for the case of too large $n_{R}$ values the artificial zeros are extremely sensitive to numerical round-off errors (in the high-precision variables) and that they change strongly, when slightly modifying the support points (e.g., a random modification $\sim 10^{-18}$ or simply changing their order in the interpolation scheme) or when changing the precise numerical algorithm (e.g., between a direct sum or Horner scheme for the evaluation of the series of the rational function). Furthermore, they do not respect the symmetry that the zeros should come in pairs of complex conjugate numbers in case of complex zeros. This is because Thiele's rational interpolation scheme breaks the symmetry due to complex conjugation once round-off errors become relevant. 

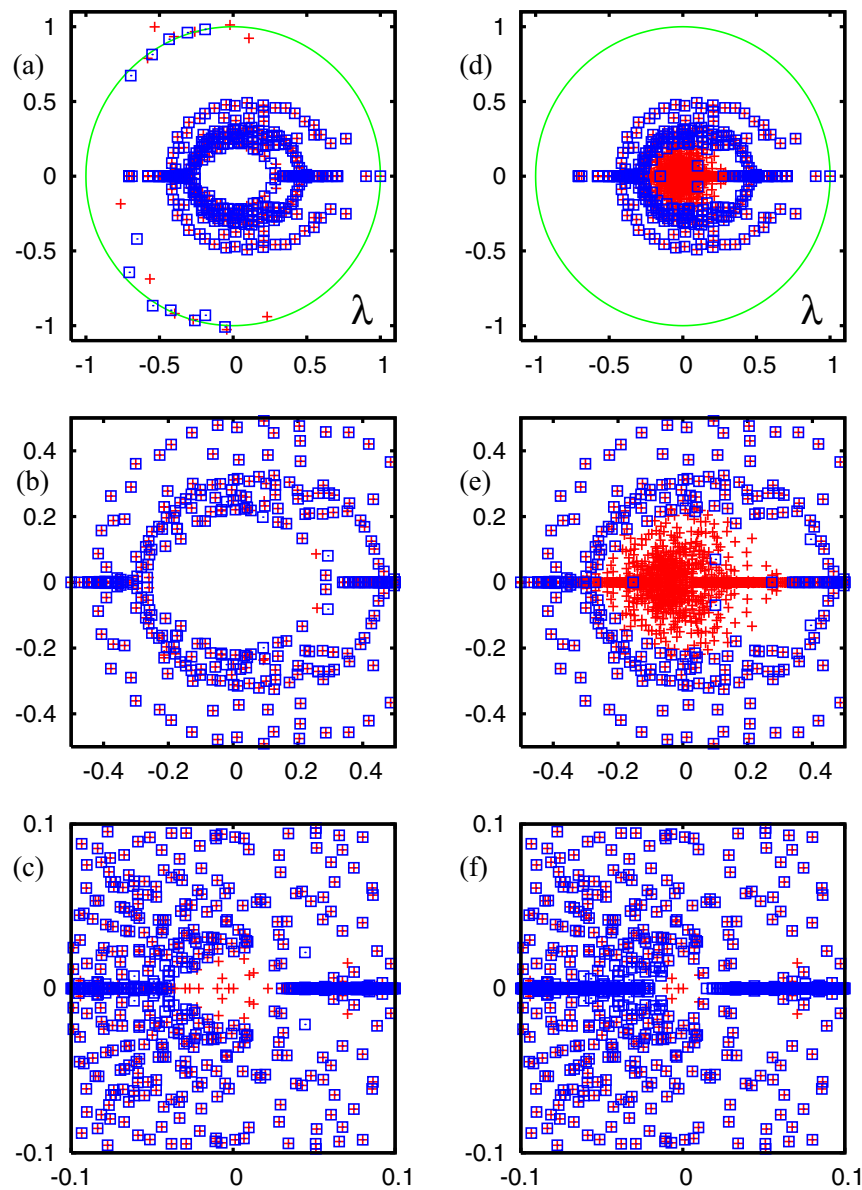

FIG. 9. (Color online) (a) Comparison of $n_{R}=340$ core space eigenvalues of $S$ for CNPR obtained by two variants of the rational interpolation method (see text) with the numerical precision of $p=1024$ binary digits, 681 support points (first variant, red/gray crosses), or 682 support points (second variant, blue/black squares). (d) Comparison of the core space eigenvalues of CNPR obtained by the high-precision Arnoldi method with $n_{A}=2000$ and $p=768$ binary digits (red/gray crosses, same data as blue/black squares in Fig. 7) with the eigenvalues obtained by (both variants of) the rational interpolation method with the numerical precision of $p=1024$ binary digits and $n_{R}=300$ eigenvalues (blue/black squares). Here both variants with 601 or 602 support points provide identical spectra (differences below $\left.10^{-14}\right)$. (b, e) Same as panels (a) and (d) with a zoomed range: $-0.5 \leqslant \operatorname{Re}(\lambda), \operatorname{Im}(\lambda) \leqslant 0.5$. (c) Comparison of the core space spectra obtained by the high-precision Arnoldi method (red/gray crosses, $n_{A}=2000$ and $p=768$ ) and by the rational interpolation method with $p=12288, n_{R}=2000$ eigenvalues (blue/black squares). (f) Same as (c) with $p=16384, n_{R}=2500$ for the rational interpolation method. Both panels (c) and (f) are shown in a zoomed range: $-0.1 \leqslant \operatorname{Re}(\lambda), \operatorname{Im}(\lambda) \leqslant 0.1$. Eigenvalues outside the shown range coincide up to graphical precision, and both variants of the rational interpolation method provide numerically identical spectra.

However, we have carefully verified that for the proper values of $n_{R}$ not being too large (e.g., $n_{R}=300$ for $p=1024$ ) the obtained zeros are numerically identical (with 52 binary digits in the final result) with respect to small changes of the support points (or their order) or with respect to different numerical algorithms and that they respect perfectly the symmetry due to complex conjugation.

This method, despite the necessity of high-precision calculations, is not very expensive, especially for the memory usage, if compared with the high-precision Arnoldi method. Furthermore, its efficiency for the computation time can be improved by the trick of summing up the largest terms in the series (D3) as a geometrical series which allows to reduce the cutoff value of $l$ by a good factor 3 , i.e., replacing $\rho_{1} \approx 0.902$ by $\rho_{2}=1 / \sqrt{2} \approx 0.707$ in the estimate (D4) of $l$ which gives $l \approx 2 p+$ const. We have increased the number of binary digits up to $p=16384$, and we find that for $p=1024,2048,4096,6144,8192,12288,16384$ we may use $n_{R}=300,500,900,1200,1500,2000,2500$ and still avoid the appearance of artificial zeros. In Fig. 9 we also compare the result of the highest precisions $p=12288$ (and $p=16384)$ using $n_{R}=2000\left(n_{R}=2500\right)$ with the high-precision Arnoldi method with $n_{A}=2000$ and $p=768$, and these spectra coincide well apart from a minor number of smallest eigenvalues. In general, the complex isolated eigenvalues converge very well (with increasing values of $p$ and $n_{R}$ ), while the strongly clustered eigenvalues on the real axis have more difficulties to converge. Comparing the results between $n_{R}=2000$ and $n_{R}=2500$ we see that the complex eigenvalues coincide on graphical precision for $|\lambda| \geqslant 0.04$ and the real eigenvalues for $|\lambda| \geqslant 0.1$. The Arnoldi method has even more difficulties on the real axis (convergence roughly for $|\lambda| \geqslant 0.15$ ) since it implicitly has to take care of the highly degenerate eigenvalues of the first group and for which it has difficulties to correctly find the degeneracies (see also Fig. 8).

In Fig. 10 we show as a summary the highest-precision spectra of $S$ with core space eigenvalues obtained by the Arnoldi method or the rational interpolation method (both at best parameter choices) and taking into account the direct subspace eigenvalues of $S$ and the above determined eigenvalues of the first group (degenerate subspace eigenvalues of $S_{0}$ ).

\section{FRACTAL WEYL LAW FOR CNPR}

The concept of the fractal Weyl law [31-33] states that the number of states $N_{\lambda}$ in a ring of complex eigenvalues with $\lambda_{c} \leqslant|\lambda| \leqslant 1$ scales in a polynomial way with the growth of matrix size:

$$
N_{\lambda}=a N^{b}
$$

where the exponent $b$ is related to the fractal dimension of underlying invariant set $d_{f}=2 b$. The fractal Weyl law was first discussed for the problems of quantum chaotic scattering in the semiclassical limit [31-33]. Later it was shown that this law also works for the Ulam matrix approximant of the Perron-Frobenius operators of dissipative chaotic systems with strange attractors [6,7]. In Ref. [11] it was established that the time growing Linux Kernel network is also characterized by the fractal Weyl law with the fractal dimension $d_{f} \approx 1.3$.

The fact that $b<1$ implies that the majority of eigenvalues drop to zero. We see that this property also appears for the CNPR if we test here the validity of the fractal Weyl law by considering a time reduced CNPR of size $N_{t}$ including the $N_{t}$ papers published until the time $t$ (measured in years) for 

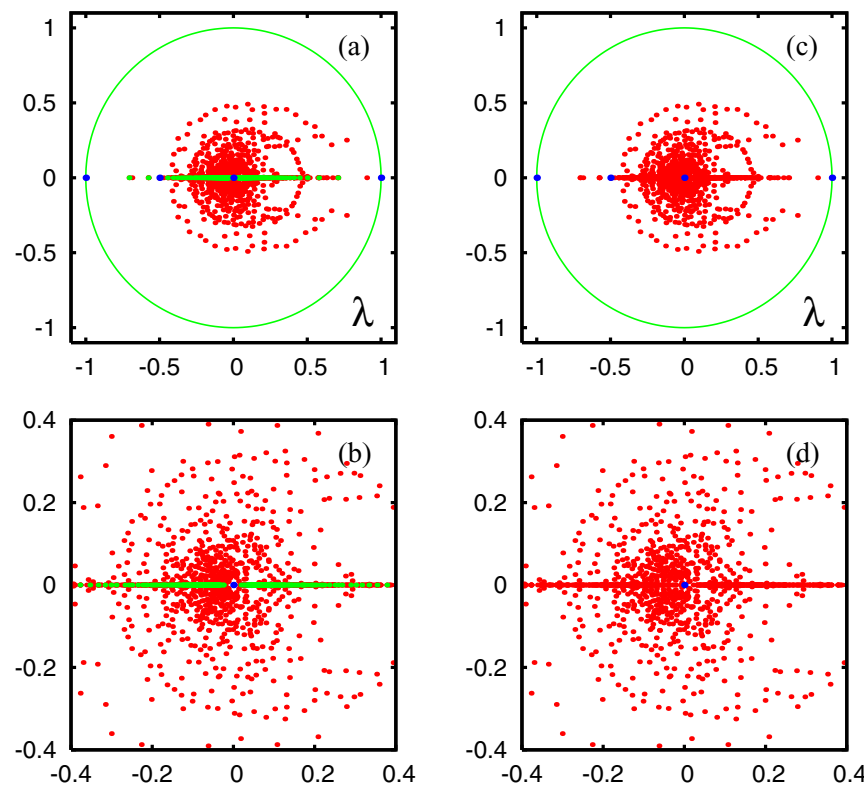

FIG. 10. (Color online) The most accurate spectrum of eigenvalues of $S$ for CNPR. (a) Red/gray dots represent the core space eigenvalues obtained by the rational interpolation method with the numerical precision of $p=16384$ binary digits, $n_{R}=2500$ eigenvalues; green (light gray) dots on the $y=0$ axis show the degenerate subspace eigenvalues of the matrix $S_{0}$, which are also eigenvalues of $S$ with a degeneracy reduced by one (eigenvalues of the first group, see text); blue/black dots show the direct subspace eigenvalues of $S$ (same as blue/black dots in left upper panel in Fig. 3). (c) Red/gray dots represent the core space eigenvalues obtained by the high-precision Arnoldi method with $n_{A}=2000$ and the numerical precision of $p=768$ binary digits, and blue dots show the direct subspace eigenvalues of $S$. Note that the Arnoldi method also determines implicitly the degenerate subspace eigenvalues of $S_{0}$, which are therefore not shown in another color. b, d) Same as in top panels (a) and (c) with a zoomed range: $-0.4 \leqslant \operatorname{Re}(\lambda), \operatorname{Im}(\lambda) \leqslant 0.4$.

different times $t$ in order to obtain a scaling behavior of $N_{\lambda}$ as a function of $N_{t}$. The data presented in Fig. 11 show that the network size grows approximately exponentially as $N_{t}=$ $2^{\left(t-t_{0}\right) / \tau}$ with the fit parameters $t_{0}=1791, \tau=11.4$. The time interval considered in Fig. 11 is $1913 \leqslant t \leqslant 2009$ since the first data point corresponds to $t=1913$ with $N_{t}=1500$ papers published between 1893 and 1913. The results for $N_{\lambda}$ show that its growth is well described by the relation $N_{\lambda}=a\left(N_{t}\right)^{b}$ for the range when the number of articles becomes sufficiently large $3 \times 10^{4} \leqslant N_{t}<5 \times 10^{5}$. This range is not very large, and probably due to that there is a certain dependence of the exponent $b$ on the range parameter $\lambda_{c}$. At the same time we note that the maximal matrix size $N$ studied here is probably the largest one used in numerical studies of the fractal Weyl law. We have $0.47<b<0.6$ for all $\lambda_{c} \geqslant 0.4$ that is definitely smaller than unity, and thus the fractal Weyl law is well applicable to the CNPR. The value of $b$ increases up to 0.7 for the data points with $\lambda_{c}<0.4$, but this is due to the fact the here $N_{\lambda}$ also includes some numerically incorrect eigenvalues related to the numerical instability of the Arnoldi method at standard double precision ( 52 binary digits) as discussed at the beginning of the previous section.
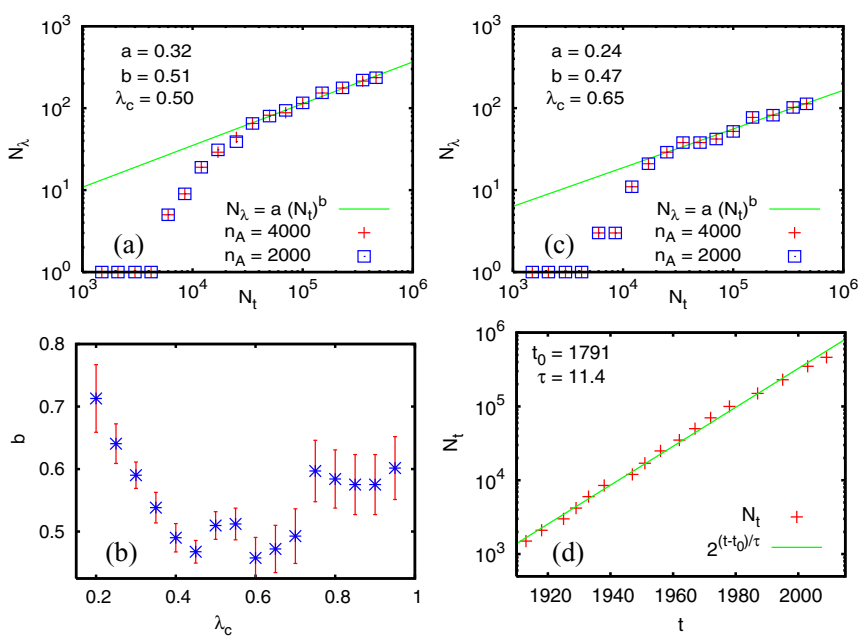

FIG. 11. (Color online) Data for the whole CNPR at different moments of time. (a) [or (c)] Number $N_{\lambda}$ of eigenvalues with $\lambda_{c} \leqslant \lambda \leqslant 1$ for $\lambda_{c}=0.50$ (or $\lambda_{c}=0.65$ ) versus the effective network size $N_{t}$ where the nodes with publication times after a cut time $t$ are removed from the network. The green/gray line shows the fractal Weyl law $N_{\lambda}=a\left(N_{t}\right)^{b}$ with parameters $a=0.32 \pm 0.08$ $(a=0.24 \pm 0.11)$ and $b=0.51 \pm 0.02(b=0.47 \pm 0.04)$ obtained from a fit in the range $3 \times 10^{4} \leqslant N_{t}<5 \times 10^{5}$. The number $N_{\lambda}$ includes both exactly determined invariant subspace eigenvalues and core space eigenvalues obtained from the Arnoldi method with double precision (52 binary digits) for $n_{A}=4000$ (red/gray crosses) and $n_{A}=2000$ (blue/black squares). (b) Exponent $b$ with error bars obtained from the fit $N_{\lambda}=a\left(N_{t}\right)^{b}$ in the range $3 \times 10^{4} \leqslant N_{t}<$ $5 \times 10^{5}$ versus cut value $\lambda_{c}$. (d) Effective network size $N_{t}$ versus cut time $t$ (in years). The green/gray line shows the exponential fit $2^{\left(t-t_{0}\right) / \tau}$ with $t_{0}=1791 \pm 3$ and $\tau=11.4 \pm 0.2$ representing the number of years after which the size of the network (number of papers published in all Physical Review journals) is effectively doubled.

We think that the most appropriate choice for the description of the data is obtained at $\lambda_{c}=0.4$, which from one side excludes small, partly numerically incorrect, values of $\lambda$ and on the other side gives sufficiently large values of $N_{\lambda}$. Here we have $b=0.49 \pm 02$ corresponding to the fractal dimension $d=0.98 \pm 0.04$. Furthermore, for $0.4 \leqslant \lambda_{c} \leqslant 0.7$ we have a rather constant value $b \approx 0.5$ with $d_{f} \approx 1.0$. Of course, it would be interesting to extend this analysis to a larger size $N$ of CNPR, but for that we still should wait about 10 years until the network size will be doubled compared to the size studied here.

\section{PROPERTIES OF EIGENVECTORS}

The results for the eigenvalue spectra of CNPR presented in the previous sections show that most of the visible eigenvalues on the real axis (except for the largest one) in Figs. 9 and 10 are due to the effect of future citations. They appear either directly due to $2 \times 2$ subblocks of the type (C2) with a cycle where two papers mutually cite each other giving the degenerate eigenvalues of the first group, or indirectly by eigenvalues of the second group, which are also numerous on the real axis. On the other hand, as can be seen in Fig. 6, for the triangular CNPR, where all future citations are removed, there 
is only the leading eigenvalue $\lambda=1$ and a small number of negative eigenvalues with $-0.27<\lambda<0$ on the real axis. All other eigenvalues are complex, and a considerable number of the largest ones are relatively close to corresponding complex eigenvalues for the whole CNPR with future citations.

The appearance of future citations is quite specific and is not a typical situation for citation networks. Therefore we consider the eigenvectors of complex eigenvalues for the triangular CNPR, which indeed represent the typical physical situation without future citations. There is no problem in evaluating these eigenvectors by the Arnoldi method, either with double precision, provided the eigenvalue of the eigenvector is situated in the region of numerically accurate eigenvalues, or with the high-precision variant of the Arnoldi method. However, for the triangular CNPR we have, according to the semianalytical theory presented above, the explicit formula

$$
\psi \propto\left(\lambda \mathbb{1}-S_{0}\right)^{-1} e / N=\sum_{j=0}^{l-1} \lambda^{-(1+j)} S_{0}^{j} e / N
$$

where the normalization is given by $\sum_{i}|\psi(i)|=1$. This expression is quite convenient, and we verified that it provides the same eigenvectors (up to numerical errors) as the Arnoldi method.

In Fig. 12 we show two eigenvectors of $S$ : one $\psi_{0}$ for the leading eigenvalue $\lambda_{0}=1$ and another $\psi_{39}$ for a complex eigenvalue at $\left|\lambda_{39}\right|<1$. The eigenvector of $\lambda_{0}$ gives the PageRank probability for the triangular CNPR (at $\alpha=1$ ). We also consider the eigenvector for the complex eigenvalue $\lambda_{39}=-0.3738799+i 0.2623941$ (eigenvalues are ordered by their absolute values starting from $\left.\lambda_{0}=1\right)$. In this figure the modulus of $\left|\psi_{j}\left(N_{t}\right)\right|$ is shown versus the time index $N_{t}$ as introduced in Fig. 11. We also indicate the positions of five famous papers: BCS 1957 [34] at $K=6$, Anderson 1958 [35] $K=63$, Benettin et al. 1976 [36] $K=441$, Thouless 1977 [37] $K=256$, and Abrahams et al. 1979 [38] $K=74$. In the first eigenvector for $\lambda_{0}=1$ all of these papers have quite dominating positions, especially BCS 1957 and Abrahams et al. 1979, which are the most important ones if compared to papers of comparable publication date. Only considerably older papers have higher positions in this vector.
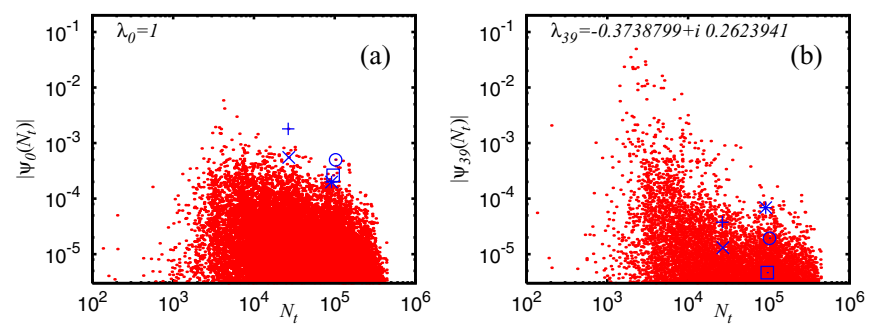

FIG. 12. (Color online) Two eigenvectors of the matrix $S$ for the triangular CNPR. Both panels show the modulus of the eigenvector components $\left|\psi_{j}\left(N_{t}\right)\right|$ versus the time index $N_{t}$ (as used in Fig. 11) with nodes/articles ordered by the publication time (small red/gray dots). The blue/black points represent five particular articles: BCS 1957 (+), Anderson $1958(\times)$, Benettin et al. $1976(*)$, Thouless $1977(\square)$, and Abrahams et al. $1979(\odot)$. The left (right) panel corresponds to the real (complex) eigenvalue $\lambda_{0}=1\left(\lambda_{39}=-0.3738799+i 0.2623941\right)$.
For the second eigenvector with complex eigenvalue the older papers (with $10^{3}<N_{t}<10^{4}$ corresponding to publications times between 1910 and 1940) are strongly enhanced in its importance, while the above five famous papers lose their importance. The top three positions of largest amplitude $\left|\psi_{39}(i)\right|$ correspond to DOI 10.1103/PhysRev.14.409 (1919), 10.1103/PhysRev.8.561 (1916), and 10.1103/PhysRev.24.97 (1917). These old articles study the radiating potentials of nitrogen, ionization impact in gases, and the abnormal low voltage arc, respectively. It is clear that this eigenvector selects a certain community of old articles related to a certain older field of interest. This fact is in agreement with the studies of eigenvectors of Wikipedia network [13] showing that the eigenvectors with $0<|\lambda|<1$ select specific communities.

It is interesting to note that the top node of the vector $\psi_{0}$ appears in the position $K_{39}=39$ in local rank index of the vector $\psi_{39}$ (ranking in decreasing order by modulus of $|\psi(i)|$ ). On the other side the top node of $\psi_{39}$ appears at position $K_{0}=30$ of vector $\psi_{0}$. This illustrates how different nodes contribute to different eigenvectors of $S$.

It is useful to characterize the eigenvectors by their inverse participation ratio (IPR) $\xi_{i}=\left(\sum_{j}\left|\psi_{i}(j)\right|^{2}\right)^{2} / \sum_{j}\left|\psi_{i}(j)\right|^{4}$, which gives an effective number of nodes populated by an eigenvector $\psi_{i}$ (see, e.g., Refs. [8,13]). For the above two vectors we find $\xi_{0}=20.67$ and $\xi_{39}=10.76$. This means that $\xi_{39}$ is mainly located on approximately 11 nodes. For $\xi_{0}$ this number is twice larger in agreement with data of Fig. 12, which show a clearly broader distribution comparing to $\xi_{39}$.

We also considered a few tens of eigenstates of $S$ of the whole CNPR. They are mainly located on the complex plane around the largest oval curve well visible in the spectrum (see Fig. 10 top right panel). The IPR value of these eigenstates with $|\lambda| \sim 0.4$ varies in the range $4<\xi<13$, showing that they are located on some effective quasi-isolated communities of articles. About 10 of them are related to the top article of $\psi_{39}$ shown in Fig. 12, meaning that these 10 vectors represent various linear combinations of vectors on practically the same community. In global terms, we can say that the eigenstates of $G$ are well localized since $\xi \ll N$. A similar situation was seen for the Wikipedia network [13].

Of course, in addition to $\xi$ it is also useful to consider the whole distribution of $\psi$ amplitudes over the nodes. Such consideration has been done for the Wikipedia network in Ref. [13]. For the CNPR we leave such detailed studies for further investigations.

\section{CHEIRANK VERSUS PAGERANK FOR CNPR}

The dependence of PageRank probability $P(K)$ on PageRank index $K$ is shown in Fig. 13. The results are similar to those of Refs. [20-23]. We note that the PageRank of the triangular CNPR has the same top nine articles as for the whole CNPR (both at $\alpha=0.85$ and with a slight interchanged order of positions $7,8,9)$. This confirms that the future citations produce only a small effect on the global ranking.

Following previous studies [25-27], in addition to the Google matrix $G$ we also construct the matrix $G^{*}$ following the same definition (1) but for the network with inverted direction of links. The PageRank vector of this matrix $G^{*}$ is called the CheiRank vector with probability $P^{*}\left(K_{i}^{*}\right)$ and CheiRank index 


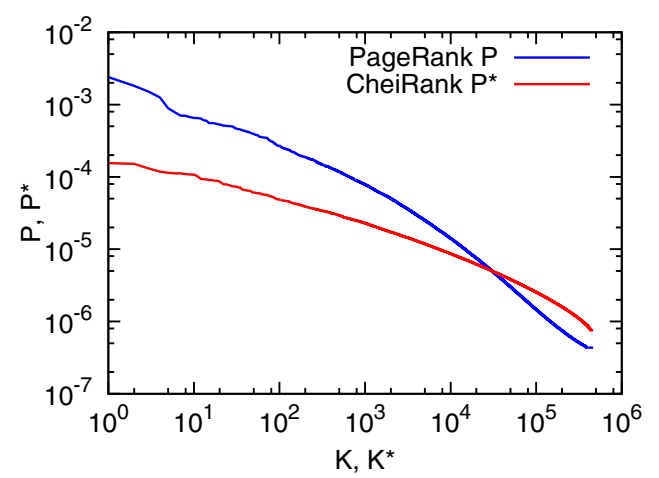

FIG. 13. (Color online) Dependence of probability of PageRank $P\left(\right.$ CheiRank $\left.P^{*}\right)$ on corresponding index $K\left(K^{*}\right)$ for the CNPR at $\alpha=0.85$.

$K^{*}$. The dependence of $P^{*}\left(K_{i}^{*}\right)$ is shown in Fig. 13. We find that the IPR values of $P$ and $P^{*}$ are $\xi=59.54$ and 1466.7, respectively. Thus $P^{*}$ is extended over significantly larger number of nodes comparing to $P$. A power law fit of the decay $P \propto 1 / K^{\beta}, P^{*} \propto 1 / K^{* \beta}$, done for a range $K, K^{*} \leqslant 2 \times 10^{5}$, gives $\beta \approx 0.57$ for $P$ and $\beta \approx 0.4$ for $P^{*}$. However, this is only an approximate description since there is a visible curvature (in a double logarithmic representation) in these distributions. The corresponding frequency distributions of ingoing links have exponents $\mu=2.87$, while the distribution of outgoing links has $\mu \approx 3.7$ for out-degree $k \geqslant 20$, even if the whole frequency dependence in this case is rather curved and a power law fit is rather approximate in this case. Thus the usual relation $\beta=1 /(\mu-1)[4,8,26]$ approximately works.

The correlation between PageRank and CheiRank vectors can be characterized by the correlator $\kappa=$ $N \sum_{i=1}^{N} P(i) P^{*}(i)-1[25,27]$. Here we find $\kappa=-0.2789$ for all CNPR, and $\kappa=-0.3187$ for CNPR without Review of Modern Physics. This is the most strong negative value of $\kappa$ among all directed networks studied previously [27]. In a certain sense the situation is somewhat similar to the Linux Kernel network where $\kappa \approx 0$ or slightly negative $(\kappa>-0.1$ [25]). For CNPR, we can say that due to an almost triangular structure of $G$ and $G^{*}$ there is a very little overlap of top ranking in $K$ and $K^{*}$ that leads to a negative correlator value, since the components $P(i) P^{*}(i)$ of the sum for $\kappa$ are small.

Each article $i$ has two indexes $K_{i}, K_{i}^{*}$ so that it is convenient to see their distribution on a 2D PageRank-CheiRank plane. The density distribution $W\left(K, K^{*}\right)=d N_{i} / d K d K^{*}$ is shown in Fig. 14. It is obtained from $100 \times 100$ cells equidistant in log scale (see details in Refs. [26,27]). For the CNPR the density is homogeneous along lines $K=-K^{*}+$ const, which corresponds to the absence of correlations between $P$ and $P^{*}$ [26,27]. For the CNPR without Review of Modern Physics we have an additional suppression of density at low $K^{*}$ values. Indeed, Review of Modern Physics contains mainly review articles with a large number of citations that place them on top of CheiRank. At the top three positions of $K^{*}$ of CNPR we have DOI 10.1103/PhysRevA.79.062512, 10.1103/PhysRevA.79.062511, and 10.1103/RevModPhys.81.1551 of 2009. These are articles with long citation lists on $K$ shell diagram $4 d$ transition elements, hypersatellites of $3 d$ transition
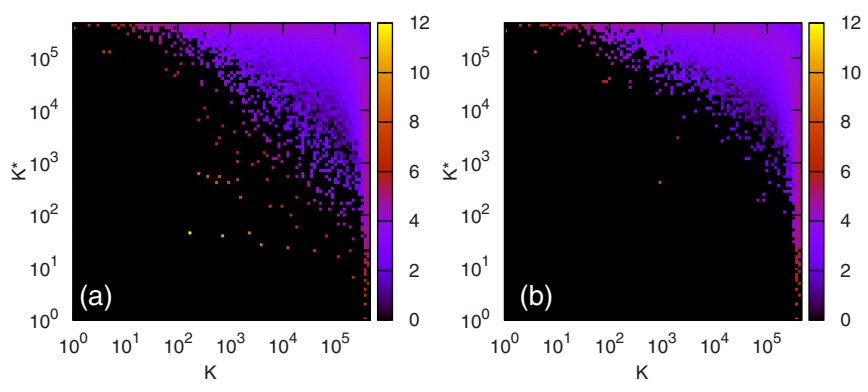

FIG. 14. (Color online) Density distribution $W\left(K, K^{*}\right)=$ $d N_{i} / d K d K^{*}$ of Physical Review articles in the PageRank-CheiRank plane $\left(K, K^{*}\right)$. Color bars show the natural logarithm of density, changing from minimal nonzero density (dark) to maximal one (white); zero density is shown by black. (a) All articles of CNPR; (b) CNPR without Rev. Mod. Phys.

metals, and superconducting phases of $f$ electron compounds, respectively. For CNPR without Review of Modern Physics the first two articles are the same, and the third one has DOI 10.1103/PhysRevB.80.224501 being about models for the coexistence of $d$ wave superconducting and charge density wave order in high-temperature cuprate superconductors. We see that the most recent articles with long citation lists are dominating.

The top PageRank articles are analyzed in detail in Refs. [20-23], and we do not discuss them here.

It is also useful to consider two-dimensional rank 2DRank $K_{2}$ defined by counting nodes in order of their appearance on ribs of squares in $\left(K, K^{*}\right)$ plane with the square size growing from $K=1$ to $N$ [26]. It selects highly cited articles with a relatively long citation list. For CNPR, we have the top three such articles with DOI 10.1103/RevModPhys.54.437 (1982), 10.1103/RevModPhys.65.851 (1993), and 10.1103/RevModPhys.58.801 (1986). Their topics are electronic properties of two-dimensional systems, pattern formation outside of equilibrium, and spin glasses facts and concepts, respectively. The first one located at $K=183, K^{*}=49$ is well visible in the left panel of Fig. 14. For CNPR without Review of Modern Physucs we find at $K_{2}=1$ the article with DOI 10.1103/PhysRevD.54.1 (1996) entitled "Review of particle physics" with much information on physical constants.

For the ranking of articles about persons in Wikipedia networks [14,26,39], PageRank, 2DRank, and CheiRank highlight in a different manner various aspects of human activity. For the CNPR, these three ranks also select different types of articles; however, due to a triangular structure of $G, G^{*}$ and absence of correlations between PageRank and CheiRank vectors, the useful side of 2DRank and CheiRank remains less evident.

\section{IMPACTRANK FOR INFLUENCE PROPAGATION}

It is interesting to quantify how an influence of a given article propagates through the whole CNPR. To analyze this property we consider the following propagator acting on an initial vector $v_{0}$ located on a given article:

$$
v_{f}=\frac{1-\gamma}{1-\gamma G} v_{0}, \quad v_{f}^{*}=\frac{1-\gamma}{1-\gamma G^{*}} v_{0} .
$$



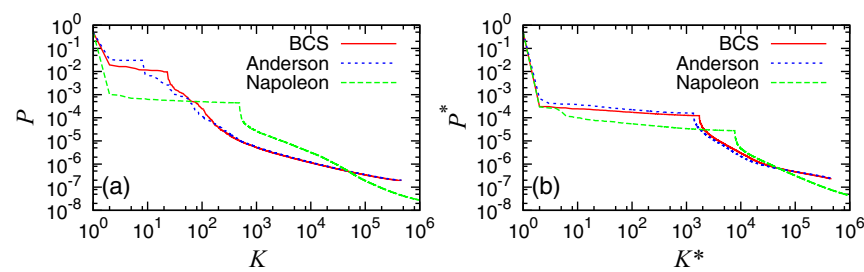

FIG. 15. (Color online) Dependence of impact vector $v_{f}$ probability $P$ and $P^{*}(\mathrm{a}, \mathrm{b})$ on the corresponding ImpactRank index $K$ and $K^{*}$ for an initial article $v_{0}$ as BCS [34] and Anderson [35] in CNPR, and Napoleon in the English Wikipedia network from Ref. [39]. Here the impact damping factor is $\gamma=0.5$.

Here $G, G^{*}$ are the Google matrices defined above, $\gamma$ is a new impact damping factor in a range $\gamma \sim 0.5-0.9$, and $v_{f}$ in the final vector generated by the propagator (7). This vector is normalized to unity $\sum_{i} v_{f}(i)=1$, and one can easily show that it is equal to the PageRank vector of a modified Google matrix given by

$$
\tilde{G}=\gamma G+(1-\gamma) v_{0} e^{T},
$$

where $e$ is the vector with unit elements. This modified Google matrix corresponds to a stochastic process where at a certain time a given probability distribution is propagated with probability $\gamma$ using the initial Google matrix $G$, and with probability $(1-\gamma)$ the probability distribution is reinitialized with the vector $v_{0}$. Then $v_{f}$ is the stationary vector from this stochastic process. Since the initial Google matrix $G$ has a similar form, $G=\alpha S+(1-\alpha) e e^{T} / N$ with the damping factor $\alpha$, the modified Google matrix can also be written as

$$
\tilde{G}=\tilde{\alpha} S+(1-\tilde{\alpha}) v_{p} e^{T}, \quad \tilde{\alpha}=\gamma \alpha,
$$

with the personalization vector [4]

$$
v_{p}=\frac{\gamma(1-\alpha) e / N+(1-\gamma) v_{0}}{1-\gamma \alpha},
$$

which is also sum normalized: $\sum_{i} v_{p}(i)=1$. Obviously similar relations hold for $G^{*}$ and $v_{f}^{*}$.

The relation (7) can be viewed as a Green function with damping $\gamma$. Since $\gamma<1$ the expansion in a geometric series is convergent, and $v_{f}$ can be obtained from about 200 terms of the expansion for $\gamma \sim 0.5$. The stability of $v_{f}$ is verified by changing the number of terms. The obtained vectors $v_{f}, v_{f}^{*}$ can be considered as effective PageRank, CheiRank probabilities $P, P^{*}$, and all nodes can be ordered in the corresponding rank index $K, K^{*}$, which we will call ImpactRank.

The results for two initial vectors located on BCS [34] and Anderson [35] articles are shown in Fig. 15. In addition we show the same probability for the Wikipedia article "Napoleon" for the the English Wikipedia network analyzed in Ref. [39]. The direct analysis of the distributions shows that the original article is located at the top position, and the next steplike structure corresponds to the articles reached by first outgoing (ingoing) links from $v_{0}$ for $G\left(G^{*}\right)$. The next visible step correspond to a second link step.

The top 10 articles for these three vectors are shown in Tables I, II, III, IV, and V. The analysis of these top articles confirms that they are closely linked with the initial article, and thus the ImpactRank gives relatively good ranking results. At the same time, some questions for such ImpactRanking still remain to be clarified. For example, in Table IV we find at the third position the well-known Review of Modern Physics article on Anderson transitions, but the paper of Abrahams et al. [38] appears only on far positions $K^{*} \approx 300$. The situation is changed if we consider all CNPR links as bidirectional, obtaining a nondirectional network. Then the paper [38] appears on the second position directly after initial article [35]. We think that such a problem appears due to triangular structure of CNPR, where there is no intersection of forward and backward flows. Indeed, for the case of Napoleon we do not see such difficulties. Thus we hope that such an approach can be applied to other directed networks.

\section{MODELS OF RANDOM PERRON-FROBENIUS MATRICES}

In this section we discuss the spectral properties of several random matrix models of Perron-Frobenius operators characterized by non-negative matrix elements and column sums normalized to unity. We call these models random Perron-Frobenius matrices (RPFM). To construct these models for a given matrix $G$ of dimension $N$ we draw $N^{2}$ independent matrix elements $G_{i j} \geqslant 0$ from a given distribution $p(G)$ [with $p(G)=0$ for $G<0$ ] with average $\langle G\rangle=1 / N$ and finite variance $\sigma^{2}=\left\langle G^{2}\right\rangle-\langle G\rangle^{2}$. A matrix obtained in this way obeys the column sum normalization only in average but not exactly for an arbitrary realization. Therefore

TABLE I. Spreading of impact of "Theory of superconductivity" paper by J. Bardeen, L. N. Cooper, and J. R. Schrieffer (doi:10.1103/PhysRev.108.1175) by Google matrix $G$ with $\alpha=0.85$ and $\gamma=0.5$.

\begin{tabular}{lcc}
\hline \hline ImpactRank & DOI & Title of paper \\
\hline 1 & $10.1103 /$ PhysRev.108.1175 & Theory of superconductivity \\
2 & $10.1103 /$ PhysRev.78.477 & Isotope effect in the superconductivity of mercury \\
3 & $10.1103 /$ PhysRev.100.1215 & Superconductivity at millimeter wave frequencies \\
4 & $10.1103 /$ PhysRev.78.487 & Superconductivity of isotopes of mercury \\
5 & $10.1103 /$ PhysRev.79.845 & Theory of the superconducting state. I. The ground ... \\
6 & $10.1103 /$ PhysRev.80.567 & Wave functions for superconducting electrons \\
7 & $10.1103 /$ PhysRev.79.167 & The hyperfine structure of Ni ${ }^{61}$ \\
8 & $10.1103 /$ PhysRev.97.1724 & Theory of the Meissner effect in superconductors \\
9 & $10.1103 /$ PhysRev.81.829 & Relation between lattice vibration and London ... \\
10 & $10.1103 /$ PhysRev.104.844 & Transmission of superconducting films ... \\
\hline \hline
\end{tabular}


TABLE II. Spreading of impact of "Absence of diffusion in certain random lattices" paper by P. W. Anderson (doi:10.1103/PhysRev.109.1492) by Google matrix $G$ with $\alpha=0.85$ and $\gamma=0.5$.

\begin{tabular}{lcc}
\hline \hline ImpactRank & DOI & Title of paper \\
\hline 1 & $10.1103 /$ PhysRev.109.1492 & Absence of diffusion in certain random lattices \\
2 & $10.1103 /$ PhysRev.91.1071 & Electronic structure of f centers: Saturation of ... \\
3 & $10.1103 /$ RevModPhys.15.1 & Stochastic problems in physics and astronomy \\
4 & $10.1103 /$ PhysRev.108.590 & Quantum theory of electrical transport phenomena \\
5 & $10.1103 /$ PhysRev.48.755 & Theory of pressure effects of foreign gases on spectral lines \\
6 & $10.1103 /$ PhysRev.105.1388 & Multiple scattering by quantum-mechanical systems \\
7 & $10.1103 /$ PhysRev.104.584 & Spectral diffusion in magnetic resonance \\
9 & $10.1103 /$ PhysRev.74.206 & A note on perturbation theory \\
10 & $10.1103 /$ PhysRev.70.460 & Nuclear induction \\
\hline \hline
\end{tabular}

we renormalize all columns to unity after having drawn the matrix elements. This renormalization provides some (hopefully small) correlations between the different matrix elements.

Neglecting these correlations for sufficiently large $N$ the statistical average of the RPFM is simply given by $\left\langle G_{i j}\right\rangle=$ $1 / N$, which is a projector matrix with the eigenvalue $\lambda=1$ of multiplicity 1 and the corresponding eigenvector being the uniform vector $e$ (with $e_{i}=1$ for all $i$ ). The other eigenvalue $\lambda=0$ is highly degenerate of multiplicity $N-1$, and its eigenspace contains all vectors orthogonal to the uniform vector $e$. Writing the matrix elements of a RPFM as $G_{i j}=\left\langle G_{i j}\right\rangle+\delta G_{i j}$ we may consider the fluctuating part $\delta G_{i j}$ as a perturbation, which only weakly modifies the unperturbed eigenvector $e$ for $\lambda=1$, but for the eigenvalue $\lambda=0$ we have to apply degenerate perturbation theory which requires the diagonalization of $\delta G_{i j}$. According to the theory of nonsymmetric real random Gaussian matrices $[5,40,41]$ it is well established that the complex eigenvalue density of such a matrix is uniform on a circle of radius $R=\sqrt{N} \sigma$ with $\sigma^{2}$ being the variance of the matrix elements. One can also expect that this holds for more general, non-Gaussian, distributions with finite variance provided that we exclude extreme long tail distribution where the typical values are much smaller than $\sigma$. Therefore we expect that the eigenvalue density of a RPFM is determined by a single parameter being the variance $\sigma^{2}$ of the matrix elements, resulting in a uniform density on a circle of radius $R=\sqrt{N} \sigma$ around $\lambda=0$, in addition to the unit eigenvalue $\lambda=1$, which is always an exact eigenvalue due to sum normalization of columns.

We now consider different variants of RPFM. The first variant is a full matrix with each element uniformly distributed in the interval $\left[0,2 / N\left[\right.\right.$, which gives the variance $\sigma^{2}=$ $1 /\left(3 N^{2}\right)$ and the spectral radius $R=1 / \sqrt{3 N}$. The second variant is a sparse RPFM matrix with $Q$ nonvanishing elements per column and which are uniformly distributed in the interval $[0,2 / Q[$. Then the probability distribution is given by $p(G)=$ $(1-Q / N) \delta(G)+(Q / N) \chi_{[0,2 / Q[}(G)$ where $\chi_{[0,2 / Q[}(G)$ is the characteristic function on the interval $[0,2 / Q$ [ (with values being 1 for $G$ in this interval and 0 for $G$ outside this interval). The average is indeed $\langle G\rangle=1 / N$, and the variance is $\sigma^{2}=4 /(3 N Q)$ (for $\left.N \gg Q\right)$ providing the spectral radius $R=2 / \sqrt{3 Q}$. We may also consider a sparse RPFM where we have exactly $Q$ nonvanishing constant elements of value $1 / Q$ in each column with random positions resulting in a variance $\sigma^{2}=1 /(N Q)$ and $R=1 / \sqrt{Q}$. The theoretical predictions for these three variants of RPFM coincide very well with numerical simulations. In Fig. 16 the complex eigenvalue spectrum for one realization of each of the three cases is shown for $N=400$ and $Q=20$, clearly confirming the circular uniform eigenvalue density with the theoretical values of $R$. We also confirm numerically the scaling behavior of $R$ as a function of $N$ or $Q$.

Motivated by the Google matrices of DNA sequences [42], where the matrix elements are distributed with a power law, we also considered a power law variant of RPFM with

TABLE III. Spreading of impact of "Theory of superconductivity" paper by J. Bardeen, L. N. Cooper, and J. R. Schrieffer (doi:10.1103/PhysRev.108.1175) by Google matrix $G^{*}$ with $\alpha=0.85$ and $\gamma=0.5$.

\begin{tabular}{lcc}
\hline \hline ImpactRank & DOI & Title of paper \\
\hline 1 & $10.1103 /$ PhysRev.108.1175 & Theory of superconductivity \\
2 & $10.1103 /$ PhysRevB.77.104510 & \\
3 & $10.1103 /$ PhysRevC.79.054328 & Temperature-dependent gap edge in strong-coupling ... \\
4 & $10.1103 /$ PhysRevB.8.4175 & Exact and approximate ensemble treatments of thermal ... \\
5 & $10.1103 /$ RevModPhys.62.1027 & Ultrasonic attenuation in superconducting molybdenum \\
6 & $10.1103 /$ PhysRev.188.737 & Properties of boson-exchange superconductors \\
7 & $10.1103 /$ PhysRev.167.361 & Transmission of far-infrared radiation through thin films ... \\
8 & $10.1103 /$ PhysRevB.77.064503 & Superconducting thin film in a magnetic field- Theory of ... \\
9 & $10.1103 /$ PhysRevB.10.1916 & Exact mesoscopic correlation functions of the Richardson ... \\
10 & $10.1103 /$ PhysRevB.79.180501 & Magnetic field attenuation by thin superconducting lead films \\
\hline \hline
\end{tabular}


TABLE IV. Spreading of impact of "Absence of diffusion in certain random lattices" paper by P. W. Anderson (doi:10.1103/PhysRev.109.1492) by Google matrix $G^{*}$ with $\alpha=0.85$ and $\gamma=0.5$.

\begin{tabular}{lcc}
\hline \hline ImpactRank & DOI & Title of paper \\
\hline 1 & $10.1103 /$ PhysRev.109.1492 & Absence of diffusion in certain random lattices \\
2 & $10.1103 /$ PhysRevA.80.053606 & Effects of interaction on the diffusion of atomic ... \\
3 & $10.1103 /$ RevModPhys.80.1355 & Anderson transitions \\
4 & $10.1103 /$ PhysRevE.79.041105 \\
5 & 10.1103 /PhysRevB.79.205120 \\
6 & $10.1103 /$ PhysRevB.80.174205 & Localization-delocalization transition in hessian ... \\
7 & $10.1103 /$ PhysRevB.80.024203 & Statistics of the two-point transmission at ... \\
8 & 10.1103 /PhysRevB.79.153104 & Localization-delocalization transitions ... \\
9 & $10.1103 /$ PhysRevB.74.104201 & Statistics of renormalized on-site energies and ... \\
10 & $10.1103 /$ PhysRevB.71.235112 & Flat-band localization in the Anderson-Falicov-Kimball model \\
\hline \hline
\end{tabular}

$p(G)=D(1+a G)^{-b}$ for $0 \leqslant G \leqslant 1$ and with an exponent $2<b<3$. The condition $G \leqslant 1$ is required because of the column sum normalization. The parameters $D$ and $a$ are determined by normalization and the average $\langle G\rangle=1 / N$. In the limit $N^{b-2} \gg 1$ we find $a \approx N /(b-2)$ and $D \approx$ $N(b-1) /(b-2)$. For $b>3$ the variance would scale with $\sim N^{-2}$ resulting in $R \sim 1 / \sqrt{N}$ as in the first variant with uniformly distributed matrix elements. However, for $b<3$ this scaling is different, and we find (for $N^{b-2} \gg 1$ )

$$
R=C(b) N^{1-b / 2}, \quad C(b)=(b-2)^{(b-1) / 2} \sqrt{\frac{b-1}{3-b}} .
$$

Figure 17 shows the results of numerical diagonalization for one realization with $N=400$ and $b=2.5$ such that we expect $R \sim N^{-0.25}$. It turns out that the circular eigenvalue density is rather well confirmed and the "theoretical radius" is indeed given by $R=\sqrt{N} \sigma$ if the variance $\sigma^{2}$ of matrix elements is determined by an average over the $N^{2}$ matrix elements of the given matrix. A study for different values of $N$ with $50 \leqslant$ $N \leqslant 2000$ also confirms the dependence $R=C N^{-\eta}$ with fit values $C=0.67 \pm 0.03$ and $\eta=0.22 \pm 0.01$. The value of $\eta=0.22$ is close to the theoretical value $1-b / 2=0.25$ but the prefactor $C=0.67$ is smaller than its theoretical value $C(2.5) \approx 1.030$. This is due to the correlations introduced by the additional column sum normalization after drawing the random matrix elements. Furthermore for the power law model with $b<3$ we should not expect a precise confirmation of the uniform circular density obtained for Gaussian distribution matrix elements. Actually, a more detailed numerical analysis of the density shows that the density for the power law model is not exactly uniform, in particular for values of $b$ close to 2 .

The important observation is that a generic RPFM (full, sparse, or with power law distributed matrix elements) has a complex eigenvalue density rather close to a uniform circle of a quite small radius (depending on the parameters $N, Q$, or $b$ ). The fact, that the realistic networks (e.g., certain university WWW networks) have Google matrix spectra very different from this [10] shows that in these networks there is indeed a subtle network structure and that slight random perturbations or variations already immediately result in uniform circular eigenvalue spectra. This was already observed in Refs. [8,9], where it was shown that certain modest random changes in the network links already provide such circular eigenvalue spectra.

We also determine the PageRank for the different variants of the RPFM, i.e., the eigenvector for the eigenvalue $\lambda=1$. It turns out that PageRank vector has practically equal probabilities on each node. This is natural since this eigenvector should be close to the uniform vector $e$, which is the "PageRank" for the average matrix $\left\langle G_{i j}\right\rangle=1 / N$. This also holds when we use a damping factor $\alpha=0.85$ for the RPFM.

Following the above discussion about triangular networks (with $G_{i j}=0$ for $i \geqslant j$ ) we also study numerically a triangular RPFM where for $j \geqslant 2$ and $i<j$ the matrix elements $G_{i j}$ are uniformly distributed in the interval $[0,2 /(j-1)[$, and for $i \geqslant j$ we have $G_{i j}=0$. Then the first column is empty, which means it corresponds to a dangling node and it needs to be replaced by $1 / N$ entries. For the triangular RPFM the situation

TABLE V. Spreading of impact of the article "Napoleon" in English Wikipedia by Google matrix $G$ and $G^{*}$ with $\alpha=0.85$ and $\gamma=0.5$.

\begin{tabular}{lcc}
\hline \hline ImpactRank & Articles $(G$ case $)$ & Articles $\left(G^{*}\right.$ case $)$ \\
\hline 1 & Napoleon & Napoleon \\
2 & French Revolution & List of orders of battle \\
3 & France & Lists of state leaders by year \\
4 & First French Empire & Names inscribed under the Arc de Triomphe \\
5 & Napoleonic Wars & List of battles involving France \\
6 & French First Republic & Order of battle of the Waterloo Campaign \\
7 & Saint Helena & Napoleonic Wars \\
8 & French Consulate & Wagram order of battle \\
9 & French Directory & Departments of France \\
10 & National Convention & Jena-Auerstedt Campaign Order of Battle \\
\hline \hline
\end{tabular}



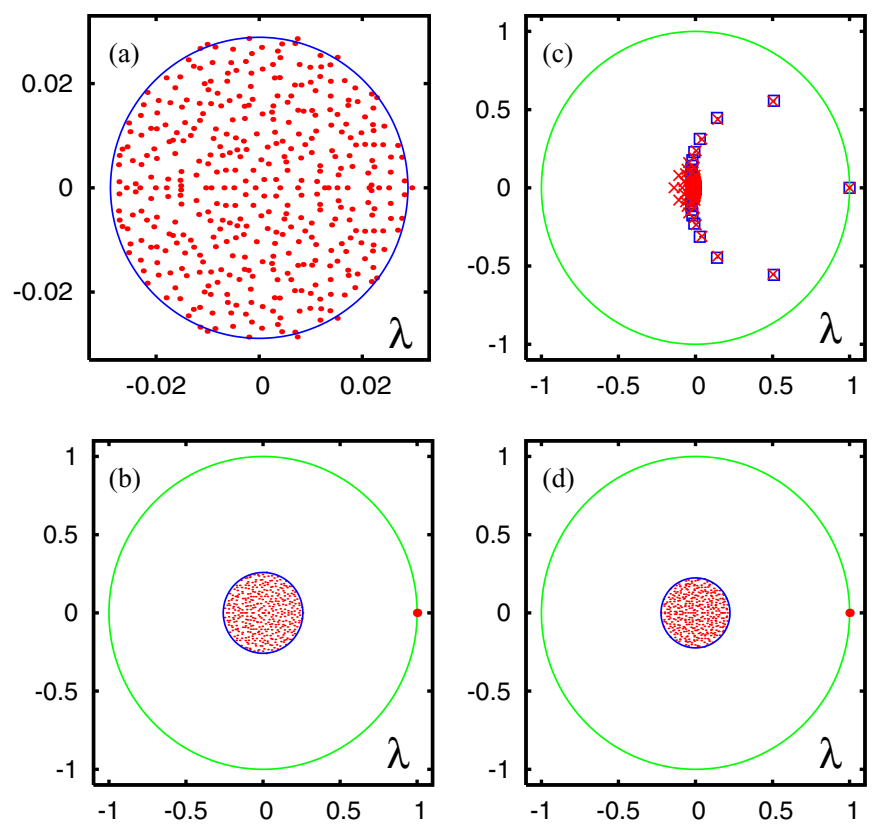

FIG. 16. (Color online) (a) Spectrum (red/gray dots) of one realization of a full uniform RPFM with dimension $N=400$ and matrix elements uniformly distributed in the interval $[0,2 / N[$; the blue/black circle represents the theoretical spectral border with radius $R=1 / \sqrt{3 N} \approx 0.02887$. The unit eigenvalue $\lambda=1$ is not shown due to the zoomed presentation range. (c) Spectrum of one realization of triangular RPFM (red/gray crosses) with nonvanishing matrix elements uniformly distributed in the interval $[0,2 /(j-1)[$ and a triangular matrix with nonvanishing elements $1 /(j-1)$ (blue/black squares); here $j=2,3, \ldots, N$ is the index number of nonempty columns and the first column with $j=1$ corresponds to a dangling node with elements $1 / N$ for both triangular cases. (b, d) Complex eigenvalue spectrum (red/gray dots) of a sparse RPFM with dimension $N=400$ and $Q=20$ nonvanishing elements per column at random positions. Panel (b) [or (d)] corresponds to the case of uniformly distributed nonvanishing elements in the interval $[0,2 / Q[$ (constant nonvanishing elements being $1 / Q$ ); the blue/black circle represents the theoretical spectral border with radius $R=2 / \sqrt{3 Q} \approx 0.2582(R=1 / \sqrt{Q} \approx 0.2236)$. In panels (b) and (d) $\lambda=1$ is shown by a larger red dot for better visibility. The unit circle is shown by green/gray line [panels (b), (c), and (d)].

changes completely since here the average matrix $\left\langle G_{i j}\right\rangle=$ $1 /(j-1)$ (for $i<j$ and $j \geqslant 2$ ) has already a nontrivial structure and eigenvalue spectrum. Therefore the argument of degenerate perturbation theory which allowed us to apply the results of standard full nonsymmetric random matrices does not apply here. In Fig. 16 one clearly sees that for $N=400$ the spectra for one realization of a triangular RPFM and its average are very similar for the eigenvalues with large modulus but both do not have at all a uniform circular density in contrast to the RPRM models without the triangular constraint discussed above. For the triangular RPFM the PageRank behaves as $P(K) \sim 1 / K$ with the ranking index $K$ being close to the natural order of nodes $\{1,2,3, \ldots\}$, which reflects the fact that the node 1 has the maximum of $N-1$ incoming links and so on.
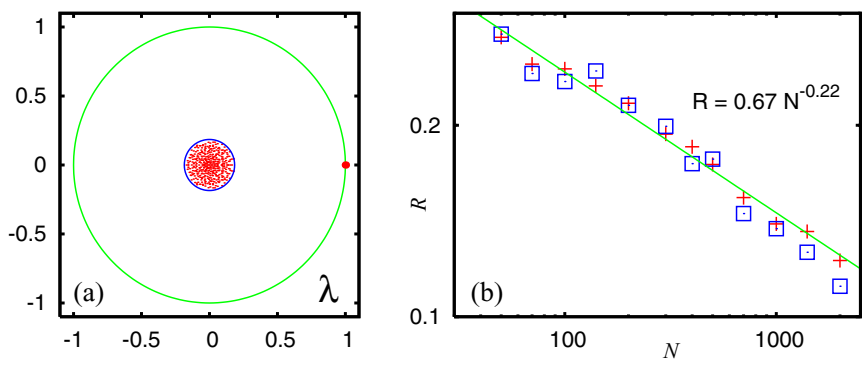

FIG. 17. (Color online) (a) Spectrum (red/gray dots) of one realization of the power law RPFM with dimension $N=400$ and decay exponent $b=2.5$ (see text); the unit eigenvalue $\lambda=1$ is shown by a large red/gray dot, the unit circle is shown by a green/gray curve; the blue/black circle represents the spectral border with theoretical radius $R=\approx 0.1850$ (see text). (b) Dependence of the spectrum border radius on matrix size $N$ for $50 \leqslant N \leqslant 2000$; red/gray crosses represent the radius obtained from theory (see text); blue/black squares correspond to the spectrum border radius obtained numerically from a small number of eigenvalues with maximal modulus; the green/gray line shows the fit $R=C N^{-\eta}$ of red/gray crosses with $C=0.67 \pm 0.03$ and $\eta=0.22 \pm 0.01$.

The study of above models shows that it is not so simple to find a good RPFM model which reproduces a typical spectral structure of real directed networks.

\section{DISCUSSION}

In this study we presented a detailed analysis of the spectrum of the CNPR for the period 1893-2009. It happens that the numerical simulations should be done with a high accuracy (up to $p=16384$ binary digits for the rational interpolation method or $p=768$ binary digits for the high-precision Arnoldi method) to determine correctly the eigenvalues of the Google matrix of CNPR at small eigenvalues $\lambda$. Due to the time ordering of citations, the CNPR $G$ matrix is close to the triangular form with a nearly nilpotent matrix structure. We show that special semianalytical methods allow us to determine efficiently the spectrum of such matrices. The eigenstates with large modulus of $\lambda$ are shown to select specific communities of articles in certain research fields, but there is no clear way on how to identify a community one is interested in.

The obtained results show that the spectrum of CNPR is characterized by the fractal Weyl law with the fractal dimension $d_{f} \approx 1$ and the growth exponent $b \approx 0.5$ being significantly smaller than unity. We think that the Physical Review network has a structure which is typical for other citation networks, and thus our result shows that the fractal Weyl law is a typical feature of citation networks.

The ranking of articles is analyzed with the help of PageRank and CheiRank vectors corresponding to forward and backward citation flows in time. It is shown that the correlations between these two vectors are small and even negative, which is similar to the case of Linux Kernel networks [27] and significantly different from networks of universities and Wikipedia. The 2DRanking on the PagRank-CheiRank plane allows us to select articles which efficiently redistribute information flow on the CNPR. 
To characterize the local impact propagation for a given article we introduce the concept of ImpactRank, which efficiently determines its domain of influence.

Finally we perform the analysis of several models of RPFM showing that such full random matrices are very far from the realistic cases of directed networks. Random sparse matrices with a limited number $Q$ of links per nodes seem to be closer to typical Google matrices concerning the matrix structure. However, such random models give a rather uniform eigenvalue density with a spectral radius $\sim 1 / \sqrt{Q}$ and a flat PageRank distribution. Furthermore they do not capture the existence of quasi-isolated communities, which generates a quasidegenerate spectrum at $\lambda=1$. Further development of RPFM models is required to reproduce the spectral properties of real modern directed networks.

In summary, we developed powerful numerical methods which allowed us to determine numerically the exact eigenvalues and eigenvectors of the Google matrix of Physical Review. We demonstrated that this matrix is close to triangular matrices of large size where numerical errors can significantly affect the eigenvalues. We show that the techniques developed in this work allow us to resolve such difficulties and obtain the exact spectrum in a semianalytical manner. The eigenvectors of eigenvalues with $|\lambda|<1$ are located on certain communities of articles related to specific scientific research subjects. We point that the random matrix models of Google matrices are still awaiting their detailed development. Indeed, matrices with random elements have a spectrum being very different from the real one. Thus, while the random matrix theory of Hermitian and unitary matrices has been very successful (see, e.g., Ref. [5]), a random matrix theory for Google matrices still waits its development. It is possible that the case of triangular matrices, which is rather similar to our CNPR case, can be a good starting point for development of such models.

\section{ACKNOWLEDGMENTS}

We thank the American Physical Society for letting us use their citation database for Physical Review [16]. This research is supported in part by the EC FET Open project "New tools and algorithms for directed network analysis" (NADINE No. 288956). This work was granted access to the HPC resources of CALMIP (Toulouse) under the allocation 2012-P0110.

\section{APPENDIX A: THEORY OF TRIANGULAR ADJACENCY MATRICES}

Let us briefly mention the analytical theory of Ref. [19] for pure triangular networks with a nilpotent matrix $S_{0}$ such that $S_{0}^{l}=0$. For integers [19] the adjacency matrix is defined as $A_{m n}=k$ where $k$ is a multiplicity defined as the largest integer such that $m^{k}$ is a divisor of $n$ and if $1<m<n$, and $k=0$ if $m=1$ or $m=n$ or if $m$ is not a divisor of $n$. Thus, we have $k=0$ if $m$ is not a divisor of $n$ and $k \geqslant 1$ if $m$ is a divisor of $n$ different from 1 and $n$. The total size $N$ of the matrix is fixed by the maximal considered integer. Then the Google matrix is constructed from $A_{m n}$ following the standard rules described above. This network of integers gives an important example of a triangular Google matrix with similar features also appearing in the Physical Review citation network. Below we discuss the general properties of such matrices.

For this we define the coefficients:

$$
c_{j}=d^{T} S_{0}^{j} e / N, \quad b_{j}=e^{T} S_{0}^{j} e / N,
$$

which are nonzero only for $j=0,1, \ldots, l-1$. The fact that the nonvanishing columns of $S_{0}$ are sum normalized and that the other columns (corresponding to dangling nodes) are zero can be written as $e^{T} S_{0}=e^{T}-d^{T}$ implying $d^{T}=e^{T}\left(\mathbb{1}-S_{0}\right)$. Using this identity and the fact that $S_{0}^{k}=0$ for $k \geqslant l$ we find

$$
\sum_{k=j}^{l-1} c_{k}=d^{T}\left(\mathbb{1}-S_{0}\right)^{-1} S_{0}^{j} e / N=e^{T} S_{0}^{j} e / N=b_{j},
$$

and in particular for $j=0$ we obtain the sum rule $\sum_{k=0}^{l-1} c_{k}=1$ and for $j=l-1$ the identity $b_{l-1}=c_{l-1}$.

Consider now a right eigenvector $\psi$ of $S$ with eigenvalue $\lambda$. If $d^{T} \psi=0$ we find from (4) that $\psi$ is also an eigenvector of $S_{0}$, and since $S_{0}$ is nilpotent the eigenvalue must be $\lambda=0$. Therefore for $\lambda \neq 0$ we have necessarily $d^{T} \psi \neq 0$, and with the appropriate normalization of $\psi$ we have $d^{T} \psi=1$, which implies together with the eigenvalue equation $\psi=$ $\left(\lambda \mathbb{1}-S_{0}\right)^{-1} e / N$ where the matrix inverse is well defined for $\lambda \neq 0$. The eigenvalue is determined by the condition

$$
0=\lambda^{l}\left(1-d^{T} \psi\right)=\lambda^{l}\left(1-d^{T} \frac{\mathbb{1}}{\lambda \mathbb{1}-S_{0}} e / N\right) .
$$

Since $S_{0}$ is nilpotent we may expand the matrix inverse in a finite series, and therefore the eigenvalue $\lambda$ is the zero of the reduced polynomial of degree $l$ :

$$
\mathcal{P}_{r}(\lambda)=\lambda^{l}-\sum_{j=0}^{l-1} \lambda^{l-1-j} c_{j}
$$

where the coefficients $c_{j}$ are given by (A1). Using $d^{T}=$ $e^{T}\left(\mathbb{1}-S_{0}\right)$ we may rewrite (A3) in the form

$0=\lambda^{l}\left(1-e^{T} \frac{\mathbb{1}-S_{0}}{\lambda \mathbb{1}-S_{0}} e / N\right)=(\lambda-1) \lambda^{l} e^{T} \frac{\mathbb{1}}{\lambda \mathbb{1}-S_{0}} e / N$,

which gives another expression for the reduced polynomial:

$$
\mathcal{P}_{r}(\lambda)=(\lambda-1) \sum_{j=0}^{l-1} \lambda^{l-1-j} b_{j}
$$

using the coefficients $b_{j}$ and confirming explicitly that $\lambda=1$ is indeed an eigenvalue of $S$. The expression (A6) can also be obtained by a direct calculation from (A2) and (A4).

Since the reduced polynomial has at most $l$ zeros $\lambda_{j}$ ( $\neq 0$ since $c_{l-1}=b_{l-1} \neq 0$ ) we find that there are at most $l$ nonvanishing eigenvalues of $S$ given by these zeros. They can also be obtained as the eigenvalues of a "small" $l \times l$ matrix. To see this let us define the following set of vectors $v_{j}$ for $j=1, \ldots, l$ by $v_{j}=c_{j-1}^{-1} S_{0}^{j-1} e / N$, where we have chosen to apply the prefactor $c_{j-1}^{-1}$ to the vector $S_{0}^{j-1} e / N$ [43]. From (4) and (A1) one finds that $S v_{j}$ can be expanded in the other 
vectors $v_{k}$ as

$$
S v_{j}=\frac{c_{j}}{c_{j-1}} v_{j+1}+c_{0} v_{1}=\sum_{k=1}^{l} \bar{S}_{k j} v_{k},
$$

where $\bar{S}_{k j}$ are the matrix elements of the $l \times l$ representation matrix

$$
\bar{S}=\left(\begin{array}{ccccc}
c_{0} & c_{0} & \cdots & c_{0} & c_{0} \\
c_{1} / c_{0} & 0 & \ldots & 0 & 0 \\
0 & c_{2} / c_{1} & \ldots & 0 & 0 \\
\vdots & \vdots & \ddots & \vdots & \vdots \\
0 & 0 & \cdots & c_{l-1} / c_{l-2} & 0
\end{array}\right) .
$$

Note that for the last vector $v_{l}$ we have $S v_{l}=c_{0} v_{1}$ since $c_{l}=0$, and therefore the matrix $\bar{S}$ provides a closed and mathematically exact representation of $S$ on the $l$-dimensional subspace generated by $v_{1}, \ldots, v_{l}$. Furthermore one can easily verify (by a recursive calculation in $l$ ) that the characteristic polynomial of $\bar{S}$ coincides with the reduced polynomial (A4). Therefore numerical diagonalization of $\bar{S}$ provides an alternative method to compute the nonvanishing eigenvalues of $S$. In principle one can also determine directly the zeros of the reduced polynomial by the Newton-Maehly method, and in Ref. [19] this was indeed done for cases with very modest values of $l \leqslant 29$. However, here for the triangular CNPR we have $l=352$ and the coefficients $c_{j}$ become very small, especially $c_{l-1} \approx 3.6 \times 10^{-352}$, a number which is (due to the exponent) outside the range of 64-bit standard double-precision numbers (IEEE 754) with 52 bits for the mantissa, 10 bits for the exponent (with respect to 2), and two bits for the signs of mantissa and exponent. This exponent range problem is not really serious and can, for example, be circumvented by a smart reformulation of the algorithm to evaluate the ratio $\mathcal{P}_{r}(\lambda) / \mathcal{P}_{r}^{\prime}(\lambda)$ using only ratios $c_{j} / c_{j-1}$, which do not have this exponent range problem. However, it turns out that in this approach the convergence of the Newton-Maehly method using double-precision arithmetic is very bad for many zeros and does not provide reliable results. In Appendix B we show how this problem can be solved using high-precision calculations, but we mention that one may also try another approach by diagonalizing numerically the representation matrix $\bar{S}$ given in (A8), which also depends on the ratios $c_{j} / c_{j-1}$.

\section{APPENDIX B: EFFECTS OF NUMERICAL ERRORS AND HIGH-PRECISION COMPUTATIONS}

We note that the Arnoldi method determines an orthonormal set of vectors $\zeta_{1}, \zeta_{2}, \zeta_{3}, \ldots, \zeta_{n_{A}}$ where the first vector $\zeta_{1}$ is obtained by normalizing a given initial vector and $\zeta_{j+1}$ is obtained by orthonormalizing $S \zeta_{j}$ to the previous vectors determined so far. It is obvious due to (A7) that for the initial uniform vector $e$ each $\zeta_{j}$ is given by a linear combination of the vectors $v_{k}$ with $k=1, \ldots, j$. Since the subspace of $v_{k}$ for $k=1, \ldots, l$ is closed with respect to applications of $S$ the Arnoldi method should, in theory, break off at $n_{A}=l$ with a zero coupling element. The latter is given as the norm of $S \zeta_{l}$ othogonalized to $\zeta_{1}, \ldots, \zeta_{l}$, and if this norm vanishes the vector $\zeta_{l+1}$ cannot be constructed and the Arnoldi method has completely explored an $S$-invariant subspace of dimension $l$.

However, due to a strong effect of round-off errors and the fact that the vectors $v_{j}$ are numerically "nearly" linearly dependent the last coupling element does not vanish numerically (when using double precision) and the Arnoldi method produces a cloud of numerically incorrect eigenvalues due to the Jordan blocks, which are mathematically outside the representation space (defined by the vectors $v_{j}$ ) but which are still explored due to round-off errors and clearly visible in Fig. 5. The double-precision spectrum of $\bar{S}$ seems to provide well-defined eigenvalues in the range where the Arnoldi method produces the "Jordan block cloud," but outside this cloud both spectra coincide only partly, mainly for the eigenvalues with a largest modulus and positive real part. For the eigenvalues with a negative real part there are considerable deviations. As can be seen in Fig. 6 the eigenvalues produced by the Arnoldi method at double precision are reliable provided that they are well outside the Jordan block cloud of incorrect eigenvalues. Therefore the deviations outside the Jordan block cloud show that the numerical double-precision diagonalization of the representation matrix $\bar{S}$ is not reliable as well, but here the effect of numerical errors is quite different as for the Arnoldi method, as is explained below.

In order to obtain an alternative and reliable numerical method to determine the spectrum of the triagonal CNRP we have also tried to determine the zeros of the reduced polynomial using higher-precision numbers with 80 or even 128 bits (quadruple precision), which helps to solve the (minor) exponent range problem (mentioned in Appendix A) because these formats use more bits for the exponent. However, there are indeed two other serious numerical problems. First, it turns out that in a certain range of the complex plane around $\operatorname{Re}(\lambda) \approx-0.1$ to -0.2 and $\operatorname{Im}(\lambda) \leqslant 0.1$ the numerical evaluation of the polynomial suffers in a severe way from an alternate sign problem with a strong loss of significance. Second, the zeros of the polynomial depend in a very sensitive way on the precision of the coefficients $c_{j}$ (see below). We have found that even 128-bit numbers are not sufficient to obtain all zeros with a reasonable graphical precision.

Therefore we use the very efficient GNU Multiple Precision Arithmetic Library (GMP library) [30]. With this library one has 31 bits for the exponent, and one may chose an arbitrary number of bits for the mantissa. We find that using 256 bits (binary digits) for the mantissa the complex zeros of the reduced polynomial can be determined with a precision of $10^{-18}$. In this case the convergence of the Newton-Maehly method is very nice, and we find that the sum (and product) of the complex zeros coincide with a high precision with the theoretical values $c_{0}$ [respectively: $(-1)^{l-1} c_{l-1}$ ] due to (A4). We have also tested different ways to evaluate the polynomial, such as the Horner scheme versus direct evaluation of the sum and for both methods using both expressions (A4) and (A6). It turns out that with 256 binary digits during the calculation the zeros obtained by the different variants of the method coincide very well within the required precision of $10^{-18}$. Of course, the coefficients $c_{j}$ or $b_{j}$ given by (A1) also need to be evaluated with the precision of 256 binary digits, but there is no problem of using high-precision vectors since the nonvanishing matrix elements of $S_{0}$ are rational numbers, 
which allow us to perform the evaluation of the vectors $S_{0}^{j} e / N$ with arbitrary precision. We also tested a random modification of $c_{j}$ according to $c_{j} \rightarrow c_{j}\left(1+10^{-16} X\right)$ where $X$ is a random number in the interval ] $-0.5,0.5[$. This modification gives significant differences of the order of $10^{-2}$ to $10^{-1}$ for some of the complex zeros and which are very visible in the graphical representation of the spectra. Therefore, the spectrum depends in a very sensitive way on these coefficients, and it is now quite clear that numerical double-precision diagonalization of $\bar{S}$, which depends according to (A8) on the values $c_{j}$, cannot provide accurate eigenvalues simply because the double-precision round-off errors of $c_{j}$ imply a sensitive change of eigenvalues. In particular some of the numerical eigenvalues of $\bar{S}$ differ quite strongly from the high-precision zeros of the reduced polynomial.

In order to study more precisely the effect of the numerical instability of the Arnoldi method due to the Jordan blocks we also use the GMP library to increase the numerical precision of the Arnoldi method. To be precise we implement the first part of this method, the Arnold iteration, in which the $n_{A} \times n_{A}$ Arnoldi representation matrix is determined by the Gram-Schmidt orthogonalization procedure, using highprecision numbers, while for the second step, the numerical diagonalization of this representation matrix, we keep the standard double precision. It turns that only the first step is numerically critical. Once the Arnoldi representation matrix is obtained in a careful and precise way, it is numerically well conditioned, and its numerical diagonalization works well with only double precision.

\section{APPENDIX C: THEORY OF DEGENERATE EIGENVALUES}

In order to understand the mechanism of the degenerate core space eigenvalues visible in Fig. 8 we extend the argumentation of Appendix A for triangular CNPR to the case of nearly triangular networks. Consider again the matrix $S$ given by Eq. (4), but now $S_{0}$ is not nilpotent. There are two groups of eigenvectors $\psi$ of $S$ with eigenvalue $\lambda$. The first group is characterized by the orthogonality $d^{T} \psi=0$ of the eigenvector $\psi$ with respect to the dangling vector $d$, and the second group is characterized by the nonorthogonality $d^{T} \psi \neq 0$. In the following, we describe efficient methods to determine all eigenvalues of the first group and a considerable number of eigenvalues of the second group. We note that for the case of a purely triangular network the first group contains only eigenvectors for the eigenvalue 0 , and the second group contains the eigenvectors for the $l$ nonvanishing eigenvalues as discussed in Appendix A. In principle there are also complications due to generalized eigenvectors (associated to nontrivial Jordan blocks), but they appear mainly for the eigenvalue zero, and for the moment we do not discuss these complications.

First, we note that the subspace eigenvectors of $S$ belong to the first group because the nodes of the subspaces of $S$ cannot contain dangling nodes, which, by construction of $S$, are linked to any other node and therefore belong to the core space. Since any subspace eigenvector $\psi$ has nonvanishing values only for subspace nodes being different from dangling nodes we have obviously $d^{T} \psi=0$. We also note that an eigenvector of $S$ of the first group with $d^{T} \psi=0$ is due to (4) also an eigenvector of $S_{0}$ with the same eigenvalue.

For the remaining eigenvectors in the first group one might try to diagonalize the matrix $S_{0}$ and check for each eigenvector of $S_{0}$ if the identity $d^{T} \psi=0$ holds, in which case we would obtain an eigenvector of $S$ of the first group but generically, and apart from the subspace eigenvectors, there is no reason that eigenvectors of $S_{0}$ with isolated nondegenerate eigenvalues obey this identity. However, if we have an eigenvalue of $S_{0}$ with a degeneracy $m \geqslant 2$ we may construct by suitable linear combinations $m-1$ linearly independent eigenvectors of $S_{0}$ which also obey $d^{T} \psi=0$, and therefore this eigenvalue with degeneracy $m$ of $S_{0}$ is also an eigenvalue with degeneracy $m-$ 1 of $S$. In order to determine the degenerate eigenvalues of $S_{0}$ it is useful to determine the subspaces of $S_{0}$, which (in contrast to the subspaces of $S$ ) may contain dangling nodes. Actually, each dangling node is a trivial invariant subspace (for $S_{0}$ ) of dimension 1 with a network matrix of size $1 \times 1$ and being zero. Explicitly we have implemented the following procedure: first, we determine the subspaces of $S$ (with 71 nodes in total) and remove these nodes from the network. Then we determine all subspaces of $S_{0}$ whose dimension is below 10. Each time such a subspace is found its nodes are immediately removed from the network. When we have tested in a first run all nodes as potential subspace nodes the procedure is repeated until no new subspaces of maximal dimension 10 are found since removal of former subspaces may have created new subspaces. Then the limit size of 10 is doubled to $20,40,80$, etc., to ensure that we do not miss large subspaces. However, for the CNPR it turns out that the limit size of 10 allows us to find all subspaces. In our procedure a subsequently found subspace may potentially have links to a former subspace leading to a block-triangular structure (and not block-diagonal structure as was done in Ref. [10]). This method to determine "relative" subspaces of a network already reduced by former subspaces is more convenient for the CNPR, which is nearly triangular, and it allows us also to determine correctly all subspace eigenvalues by diagonalizing each relative subspace network. The removal of subspace nodes of $S$ and $S_{0}$ reduces the network size from $N=463348$ to 404959 . In the next step we remove in the same way the subspaces of the transpose $S_{0}^{T}$ of $S_{0}$ (since the eigenvalues of $S_{0}^{T}$ and $S_{0}$ are the same), which reduces the network size further to 90965 . In total this procedure provides a block-triangular structure of $S_{0}$ as

$$
S_{0}=\left(\begin{array}{ccccccc}
S_{1} & * & \ldots & & & \cdots & * \\
0 & S_{2} & * & & & & \vdots \\
\vdots & \ddots & \ddots & \ddots & & & \vdots \\
& & 0 & B & * & & \\
\vdots & & & 0 & T_{1} & * & \vdots \\
\vdots & & & & 0 & T_{2} & * \\
0 & \ldots & & & \ldots & \ddots & \ddots
\end{array}\right) \text {, }
$$

where $S_{1}, S_{2}, \ldots$ represent the diagonal subblocks associated to the subspaces of $S$ and $S_{0}$ and $T_{1}, T_{2}, \ldots$ represent the 
diagonal subblocks associated to the subspaces of $S_{0}^{T}$, and $B$ is the "bulk" part for the remaining network of 90965 nodes. The stars represent potential nonvanishing entries whose values do not influence the eigenvalues of $S_{0}$. The subspace blocks $S_{1}, S_{2}, \ldots$ and $T_{1}, T_{2}, \ldots$, which are individually of maximal dimension 10 , can be directly diagonalized, and it turns that out of 372382 eigenvalues in these blocks only about 4000 eigenvalues (counting degeneracies) or 950 eigenvalues (noncounting degeneracies) are different from zero. Most of these eigenvalues are not degenerate and are therefore not eigenvalues of $S$, but there are still quite many degenerate eigenvalues at $\lambda= \pm 1 / \sqrt{n}$ with $n \geqslant 2$ taking small integer values and that are also eigenvalues of $S$ with a degeneracy reduced by one.

Concerning the bulk block $B$ we can write it in the form $B=B_{0}+f_{1} e_{1}^{T}$, where $f_{1}$ is the first column vector of $B$ and $e_{1}^{T}=(1,0, \ldots, 0)$. The matrix $B_{0}$ is obtained from $B$ by replacing its first column to zero. We can apply the above argumentation between $S$ and $S_{0}$ in the same way to $B$ and $B_{0}$; i.e., the degenerate eigenvalues of $B_{0}$ with degeneracy $m$ are also eigenvalues of $B$ with degeneracy $m-1$ (with eigenvectors obeying $e_{1}^{T} \psi=0$ ) and therefore eigenvalues of $S$ with degeneracy $m-2$. The matrix $B_{0}$ is decomposed in a similar way as in (C1) with subspace blocks, which can be diagonalized numerically, and a new bulk block $\tilde{B}$ of dimension 63559 and which may be treated in the same way by taking out its first column. This procedure provides a recursive scheme which after nine iterations stops with a final bulk block of zero size. At each iteration we keep only subspace eigenvalues with degeneracies $m \geqslant 2$ and which are joined with reduced degeneracies $m-1$ to the subspace spectrum of the previous iteration. For this joined spectrum we keep again only eigenvalues with degeneracies $m \geqslant 2$ which are joined with the subspace spectrum of the next higher level and so on.

In this way we have determined all eigenvalues of $S_{0}$ with a degeneracy $m \geqslant 2$ which belong to the eigenvalues of $S$ of the first group. Including the direct subspace of $S$ there are 4999 nonvanishing eigenvalues (counting degeneracies) or 442 nonvanishing eigenvalues (noncounting degeneracies). The degeneracy of the zero eigenvalue (or the dimension of the generalized kernel) is found by this procedure to be 455789 , but this would only be correct assuming that there are no general eigenvectors of higher order (representation vectors of nontrivial Jordan blocks), which is clearly not the case. The Jordan subspace structure of the zero eigenvalue complicates the argumentation. Here at each iteration step the degeneracy has to be reduced from $m$ to $m-D$ where $D>1$ is the dimension of the maximal Jordan block since each generalized eigenvector at a given order has to be treated as an independent vector when constructing vectors obeying the orthogonality with respect to the dangling vector $d$. Therefore the degeneracy of the zero eigenvalue cannot be determined exactly, but we may estimate its degeneracy of about $\sim 455000$ out of 463348 nodes in total. This implies that the number of nonvanishing eigenvalues is about $\sim 8000-9000$, which is considerably larger than the value of 352 for the triangular CNPR but still much smaller than the total network size.

In Table VI we provide the degeneracies for some of the eigenvalues $\pm 1 / \sqrt{n}$ for integer $n$ in the range $1 \leqslant n \leqslant 25$.
TABLE VI. Degeneracies of the eigenvalues with largest modulus for the whole CNPR whose eigenvectors $\psi$ belong to the first group and obey the orthogonality $d^{T} \psi=0$ with the dangling vector $d$.

\begin{tabular}{|c|c|}
\hline$\lambda$ & Degeneracy \\
\hline 1 & 27 \\
\hline-1 & 18 \\
\hline $\pm 1 / \sqrt{2}$ & 27 \\
\hline $\pm 1 / \sqrt{3}$ & 20 \\
\hline $1 / 2$ & 58 \\
\hline$-1 / 2$ & 52 \\
\hline $\pm 1 / \sqrt{5}$ & 20 \\
\hline $\pm 1 / \sqrt{6}$ & 52 \\
\hline $\pm 1 / \sqrt{7}$ & 6 \\
\hline $\pm 1 / \sqrt{8}$ & 44 \\
\hline $1 / 3$ & 47 \\
\hline$-1 / 3$ & 39 \\
\hline $\pm 1 / \sqrt{10}$ & 33 \\
\hline $\pm 1 / \sqrt{11}$ & 1 \\
\hline $\pm 1 / \sqrt{12}$ & 85 \\
\hline $\pm 1 / \sqrt{14}$ & 15 \\
\hline $\pm 1 / \sqrt{15}$ & 46 \\
\hline $1 / 4$ & 52 \\
\hline$-1 / 4$ & 42 \\
\hline $\pm 1 / \sqrt{18}$ & 29 \\
\hline $\pm 1 / \sqrt{20}$ & 60 \\
\hline $\pm 1 / \sqrt{21}$ & 30 \\
\hline $\pm 1 / \sqrt{22}$ & 3 \\
\hline $\pm 1 / \sqrt{24}$ & 69 \\
\hline $1 / 5^{\prime}$ & 20 \\
\hline$-1 / 5$ & 11 \\
\hline
\end{tabular}

The degeneracies for $+1 / \sqrt{n}$ and $-1 / \sqrt{n}$ are identical for nonsquare numbers $n$ (with noninteger $\sqrt{n}$ ) and different for square numbers (with integer $\sqrt{n}$ ). Apparently for nonsquare numbers the eigenvalues are only generated from effective $2 \times 2$ blocks:

$$
\left(\begin{array}{cc}
0 & 1 / n_{1} \\
1 / n_{2} & 0
\end{array}\right) \Rightarrow \lambda= \pm \frac{1}{\sqrt{n_{1} n_{2}}}
$$

with positive integers $n_{1}$ and $n_{2}$ such that $n=n_{1} n_{2}$, while for square numbers $n=m^{2}$ they may be generated by such blocks or by simple $1 \times 1$ blocks containing $1 / m$ such that the degeneracy for $+1 / \sqrt{n}=+1 / m$ is larger than the degeneracy for $-1 / \sqrt{n}=-1 / m$. Furthermore, statistically the degeneracy is smaller for prime numbers $n$ or numbers with less factorization possibilities and larger for numbers with more factorization possibilities. The Arnoldi method (with 52 binary digits for double-precision arithmetic and $n_{A}=8000$ ) provides according to the sizes of the plateaux visible in Fig. 8 the overall approximate degeneracies $\sim 60$ for $|\lambda|=1 / \sqrt{2}$ (i.e., $\pm 1 / \sqrt{2}$ counted together), $\sim 50$ for $|\lambda|=1 / \sqrt{3}$, and $\sim$ 115 for $|\lambda|=1 / 2$. These values are coherent with (but slightly larger than) the values 54, 40, and 110 taken from Table VI. Actually, as we will see below, the slight differences between the degeneracies obtained from Fig. 8 and from Table VI are 
indeed relevant and correspond to some eigenvalues of the second group which are close but not identical to $\pm 1 / \sqrt{2}$, $\pm 1 / \sqrt{3}$, or $\pm 1 / 2$ and do not contribute in Table VI.

\section{APPENDIX D: RATIONAL INTERPOLATION METHOD}

We now consider the eigenvalues $\lambda$ of $S$ for the eigenvectors of the second group with nonorthogonality $d^{T} \psi \neq 1$ or $d^{T} \psi=1$ after proper renormalization of $\psi$. Now $\psi$ cannot be an eigenvector of $S_{0}$, and $\lambda$ is not an eigenvalue of $S_{0}$. Similarly as in Appendix A the eigenvalue equation $S \psi=\lambda \psi$, the condition $d^{T} \psi=1$ and (4) imply that the eigenvalue $\lambda$ of $S$ is a zero of the rational function

$$
\mathcal{R}(\lambda)=1-d^{T} \frac{\mathbb{1}}{\lambda \mathbb{1}-S_{0}} e / N=1-\sum_{j, q} \frac{C_{j q}}{\left(\lambda-\rho_{j}\right)^{q}},
$$

where we have formally expanded the vector $e / N$ in eigenvectors of $S_{0}$ and with $\rho_{j}$ being the eigenvalues of $S_{0}$ and $q$ is the order of the eigenvector of $\rho_{j}$ used in this expansion, i.e., $q=1$ for simple eigenvectors and $q>1$ for generalized eigenvectors of higher order due to Jordan blocks. Note that even the largest possible value of $q$ for a given eigenvalue may be (much) smaller than its multiplicity $m$. Furthermore the case of simple repeating eigenvalues (with simple eigenvectors) with higher multiplicity $m>1$ leads only to several identical terms $\sim\left(\lambda-\rho_{j}\right)^{-1}$ for any eigenvector of this eigenvalue, thus all contributing to the coefficients $C_{j q}$ and whose precise values we do not need to know in the following. For us the important point is that the second identity in (D1) establishes that $\mathcal{R}(\lambda)$ is indeed a rational function whose denominator and numerator polynomials have the same degree and whose poles are (some of) the eigenvalues of $S_{0}$.

We mention that one can also show by a simple determinant calculation (similar to a calculation shown in Ref. [19] for triangular networks with nilpotent $S_{0}$ ) that

$$
P_{S}(\lambda)=P_{S_{0}}(\lambda) \mathcal{R}(\lambda),
$$

where $P_{S}(\lambda)$ [or $P_{S_{0}}(\lambda)$ ] is the characteristic polynomial of $S\left(S_{0}\right)$. Therefore those zeros of $\mathcal{R}(\lambda)$ which are not zeros of $P_{S_{0}}(\lambda)$ (i.e., not eigenvalues of $S_{0}$ ) are indeed zeros of $P_{S}(\lambda)$ (i.e., eigenvalues of $S$ ) since there are not poles of $\mathcal{R}(\lambda)$. Furthermore, generically the simple zeros $P_{S_{0}}(\lambda)$ also appear as poles in $\mathcal{R}(\lambda)$ and are therefore not eigenvalues of $S$. However, for a zero of $P_{S_{0}}(\lambda)$ (eigenvalue of $S_{0}$ ) with higher multiplicity $m>1$ (and unless $m$ is equal to the maximal Jordan block order $q$ associated to this eigenvalue of $S_{0}$ ) the corresponding pole in $\mathcal{R}(\lambda)$ only reduces the multiplicity to $m-1$ (or $m-q$ in case of higher order generalized eigenvectors), and we also have a zero of $P_{S}(\lambda)$ (eigenvalue of $S$ ). Some of the eigenvalues of $S_{0}$, whose eigenvectors $\psi$ are orthogonal to the dangling vector $\left(d^{T} \psi=0\right)$ and do not contribute in the expansion in (D1), are not poles of $\mathcal{R}(\lambda)$ and therefore also eigenvalues of $S$. This concerns essentially the direct subspace eigenvalues of $S$, which are also direct subspace eigenvalues of $S_{0}$ as already discussed in Appendix C. In total the identity (D2) confirms exactly the above picture that there are two groups of eigenvalues and with the special role of direct subspace eigenvalues belonging to the first group.

Our aim is to determine numerically the zeros of the rational function $\mathcal{R}(\lambda)$. In order to evaluate this function we expand the first identity in (D1) in a matrix geometric series, and we obtain

$$
\mathcal{R}(\lambda)=1-\sum_{j=0}^{\infty} c_{j} \lambda^{-1-j}
$$

with the coefficients $c_{j}$ defined in (A1) and provided that this series converges. In Appendix A, where we discussed the case of a nilpotent matrix $S_{0}$ with $S_{0}^{l}=0$, the series was finite, and for this particular case we had $\mathcal{R}(\lambda)=\lambda^{-l} \mathcal{P}_{r}(\lambda)$ where $\mathcal{P}_{r}(\lambda)$ was the reduced polynomial defined in (A4) and whose zeros provided the $l$ nonvanishing eigenvalues of $S$ for nilpotent $S_{0}$.

However, for the CNPR the series are infinite since all $c_{j}$ are different from zero. One may first try a crude approximation and simply replace the series by a finite sum for $j<l$ and using some rather large cutoff value for $l$ and determine the zeros in the same way as for the nilpotent case (a high-precision calculation of the zeros of the reduced polynomial of degree l). It turns that in this way we obtain correctly the largest core space eigenvalue of $S$ as $\lambda_{1}=0.999751822283878$ which is also obtained by (any variant of) the Arnoldi method. However, the other zeros obtained by this approximation lie all on a circle of radius $\approx 0.9$ in the complex plane and obviously do not represent any valid eigenvalues. Increasing the cutoff value $l$ does not help either, and it increases only the density of zeros on this circle. To understand this behavior we note that in the limit $j \rightarrow \infty$ the coefficients $c_{j}$ behave as $c_{j} \propto \rho_{1}^{j}$ where $\rho_{1}=0.902448280519224$ is the largest eigenvalue of the matrix $S_{0}$ with an eigenvector nonorthogonal to $d$. Note that the matrix $S_{0}$ also has some degenerate eigenvalues at +1 and -1 , but these eigenvalues are obtained from the direct subspace eigenvectors of $S$ (which are also direct subspace eigenvectors of $S_{0}$ ) and which are orthogonal to the dangling vector $d$ and do not contribute in the rational function (D1). It turns actually out that the eigenvalue $\rho_{1}$ is also the largest subspace space eigenvalue of $S_{0}$ (after having removed the direct subspace nodes of $S$ ). By analyzing explicitly the small-dimensional subspace related to this eigenvalue one can show that $\rho_{1}$ is given as the largest solution of the polynomial equation $x^{3}-\frac{2}{3} x-\frac{2}{15}=0$ and can therefore be expressed as $\rho_{1}=2 \operatorname{Re}\left[(9+i \sqrt{119})^{1 / 3}\right] /(135)^{1 / 3}$. The asymptotic behavior $c_{j} \propto \rho_{1}^{j}$ is also confirmed by the direct numerical evaluation of $c_{j}$. Therefore the series (D3) converges only for $|\lambda|>\rho_{1}$, and a simple (even very large) cutoff in the sum implies that only eigenvalues $\left|\lambda_{j}\right|>\rho_{1}$ can be determined as a zero of the finite sum. The only eigenvalue respecting this condition is the largest core space eigenvalue $\lambda_{1}$ given above.

One may try to improve this by a "better" approximation, which consists of evaluating the sum exactly up to some value $l$ and then to replace the remaining sum as a geometric series with the approximation $c_{j} \approx c_{l} \rho_{1}^{j-l}$ for $j \geqslant l$ and with $\rho_{1}$ determined as the ratio $\rho_{1}=c_{l} / c_{l-1}$ (which provides a sufficient approximation) or taken as its exact (high precision) value. This improved approximation results in $\mathcal{R}(\lambda) \approx$ $\lambda^{-l}\left(\lambda-\rho_{1}\right)^{-1} \mathcal{P}(\lambda)$ with a polynomial $\mathcal{P}(\lambda)$ whose zeros provide in total four correct eigenvalues. Apart from $\lambda_{1}$ it also gives $\lambda_{2}=0.902445536212661$ (note that this eigenvalue of $S$ is very close but different to the eigenvalue $\rho_{1}$ of $S_{0}$ ) and $\lambda_{3,4}=0.765857950563684 \pm i 0.251337495625571$ such that $\left|\lambda_{3,4}\right|=0.806045245100386$. All these four core space 
eigenvalues coincide very well with the first four eigenvalues obtained from the Arnoldi method. However, the other zeros of the polynomial $\mathcal{P}(\lambda)$ lie again on a circle, now with a reduced radius $\approx 0.7$, and do not coincide with eigenvalues of $S$. This can be understood by the fact that the coefficients $c_{j}$ obey for $j \rightarrow \infty$ the more precise asymptotic expression $c_{j} \approx C_{1} \rho_{1}^{j}+C_{2} \rho_{2}^{j}+C_{2} \rho_{3}^{j}+\cdots$ with the next eigenvalues $\rho_{2}=1 / \sqrt{2} \approx 0.707$ and $\rho_{3}=-\rho_{2}$. Here the first term $C_{1} \rho_{1}^{j}$ is dealt with analytically by the replacement of the geometric series, but the other terms create a new convergence problem. Therefore the improved approximation allows us only to determine the four core space eigenvalues with $\left|\lambda_{j}\right|>\left|\rho_{2,3}\right|=$ $1 / \sqrt{2}$. To obtain more valid eigenvalues it seems to be necessary to sum up by geometric series many of the next terms, not only the next two terms due to $\rho_{2}$ and $\rho_{3}$, but also the following terms of smaller eigenvalues $\rho_{j}$ of $S_{0}$. In other words the exact pole structure of the rational function $\mathcal{R}(\lambda)$ has be kept as best as possible.

Therefore due to the rational structure of the function $\mathcal{R}(\lambda)$ with many eigenvalues $\rho_{j}$ of $S_{0}$ that determine its precise pole structure we suggest the following numerical approach using high-precision arithmetic. For a given number $p$ of binary digits, e.g., $p=1024$, we determine the coefficients $c_{j}$ for $j<l$ where the cutoff value

$$
l \approx \frac{\ln \left(1-\rho_{1}\right)-p \ln (2)}{\ln \left(\rho_{1}\right)} \approx 6.753 p+\mathrm{const}
$$

is sufficiently large to evaluate the sum (D3) accurately in the given precision of $p$ binary digits (error below $2^{-p}$ ) for all complex values $\lambda$ on the unit circle, i.e., $|\lambda|=1$, where the series converges well. Furthermore we choose a number $n_{R}$ of "eigenvalues" we want to calculate, e.g., $n_{R}=300$, and evaluate the rational function $\mathcal{R}(z)$ at $n_{S}=2 n_{R}+1$ support points $z_{j}=\exp \left(2 \pi i j / n_{S}\right)\left(j=0, \ldots, n_{S}-1\right)$ uniformly distributed on the unit circle using the series (D3). Then we calculate the rational function $R_{I}(z)$ which interpolates $\mathcal{R}(z)$ at the $n_{S}$ support points $z_{j}, R_{I}\left(z_{j}\right)=\mathcal{R}\left(z_{j}\right)$, using Thiele's interpolation formula. Then the numerator and denominator polynomials of $R_{I}(z)$ are both of degree $n_{R}$. Thiele's interpolation formula expresses $R_{I}(z)$ in terms of a continued fraction expansion using inverse differences. This method is quite standard and well described in the literature of numerical mathematics; see, for example, Ref. [44]. After having evaluated a table of $n_{S}$ inverse differences (with $n_{S}^{2} / 2$ operations) one can evaluate arbitrary values of $R_{I}(z)$ using the continued fraction expansion (with $n_{S}$ operations). It is not very difficult to derive from the continued fraction expansion a recursive scheme to evaluate the values of the numerator and denominator polynomials separately as well as their derivatives. Using this scheme we determine the $n_{R}$ complex zeros of the numerator polynomial using the (high-precision variant of the) Newton-Maehly method. These zeros correspond to the zeros of the rational functional $\mathcal{R}(z)$ and are taken as approximate eigenvalues of the matrix $S$ of the second group. The main idea of this approach is to evaluate these zeros from the analytical continuation of $\mathcal{R}(z)$ using values for $|z|=1$ to determine its zeros well inside the unit circle.
We also consider a second variant of the method where the number of support points $n_{S}=2 n_{R}+2$ is even (instead of $n_{S}=2 n_{R}+1$ being odd as for the first variant). In this case the numerator polynomial is of degree $n_{R}+1$ (instead of $n_{R}$ ) while the denominator polynomial is of degree $n_{R}$, and we choose to interpolate the inverse of the rational function $1 / \mathcal{R}(z)$ [instead of $\mathcal{R}(z)$ itself] by $R_{I}(z)$ such that the zeros of $\mathcal{R}(z)$ are given by the $n_{R}$ zeros of the denominator (instead of the numerator) polynomial of $R_{I}(z)$.

The number $n_{R}$ must not be too small in order to well approximate the second identity in (D1) by the fit function. On the other hand for a given precision of $p$ binary digits the number of $n_{R}$ must not be too large as well because the coefficients $c_{j}$, which may be written as the expansion $c_{j}=\sum_{v} C_{v} \rho_{\nu}^{j}$, do not contain enough information to resolve its structure for the smaller eigenvalues $\rho_{j}$ of $S_{0}$. Therefore for too large values of $n_{R}$ (for a given precision), we obtain additional artificial zeros of the numerator polynomial (or of the denominator polynomial for the second variant) of $R_{I}(z)$, mostly close to the unit circle, somehow as additional nodes around the support points.

It turns out that for the proper combination of $p$ and $n_{R}$ values the method provides highly accurate eigenvalues and works astonishingly well. In particular for values of $n_{R}$ below a certain threshold (depending on the precision $p$ ) both variants of the method with odd or even number of support points provide numerically identical zeros (with final results rounded to 52 binary digits), which indeed coincide very accurately (for most of them) with the eigenvalues of $S$ we want to determine.

We note that the rational interpolation method allows us only to determine the eigenvalues of $S$ of the second group, i.e., the eigenvalues which are not eigenvalues of $S_{0}$ and whose eigenvectors obey $d^{T} \psi \neq 0$. The eigenvalues of the first group (with $d^{T} \psi=0$ ) have to be determined separately by the scheme of degenerate subspace eigenvalues of $S_{0}$ described in Appendix C. In particular the eigenvalues given in Table VI and belonging to the first group are not zeros of the rational function $\mathcal{R}(z)$ (they are actually poles of this function), but it turns out that there are some zeros of $\mathcal{R}(z)$ which are very close but not identical to some of the values in Table VI. For example, the rational interpolation method provides the following zeros: $1 / 2+3.13401098 \times 10^{-5}, 1 / 2+1.3279300 \times 10^{-7}$, $1 / \sqrt{2}-1.1597 \times 10^{-10}$ or $1 / \sqrt{2}-6.419004 \times 10^{-8}$, which are indeed accurate in the given precision since they are stable for all values of $p \geqslant 1024$ and the corresponding maximal value of $n_{R}$, and we have stopped the Newton iteration when the error of a zero was clearly below $10^{-18}$. These zeros are also found with the same precision in the data of the high-precision Arnoldi method for the three different values of 256, 512, or 768 binary digits. However, based only on results of the Arnoldi method it is not really clear if the small corrections to $1 / 2$ or $1 / \sqrt{2}$ are real and exact or numerically artificial since the Arnoldi method has indeed problems with degenerate and clustered eigenvalues [17]. Therefore the rational interpolation method provides an independent and strong confirmation of the accuracy of these type of eigenvalues. We attribute their existence to a quasisubspace structure, similarly as discussed in Ref. [10], with a matrix subblock as in (C2) but which is still very weakly coupled (by many indirect network links) to the core space. 
[1] S. Brin and L. Page, Comput. Netw. ISDN Syst. 30, 107 (1998).

[2] A. A. Markov, Rasprostranenie zakona bol'shih chisel na velichiny, zavisyaschie drug ot druga, Izvestiya Fizikomatematicheskogo obschestva pri Kazanskom universitete, 2-ya seriya, 15, 135 (1906) (in Russian) [English trans.: Extension of the limit theorems of probability theory to a sum of variables connected in a chain, reprinted in Appendix B of R. A. Howard, Dynamic Probabilistic Systems, Vol. 1, Markov Models (Dover Publications, New York, 2007)].

[3] M. Brin and G. Stuck, Introduction to Dynamical Systems (Cambridge University Press, Cambridge, 2002).

[4] A. M. Langville and C. D. Meyer, Google's PageRank and Beyond: The Science of Search Engine Rankings (Princeton University Press, Princeton, 2006).

[5] M. L. Mehta, Random Matrices (Elsevier-Academic Press, Amsterdam, 2004).

[6] D. L. Shepelyansky and O. V. Zhirov, Phys. Rev. E 81, 036213 (2010).

[7] L. Ermann and D. L. Shepelyansky, Eur. Phys. J. B 75, 299 (2010).

[8] O. Giraud, B. Georgeot, and D. L. Shepelyansky, Phys. Rev. E 80, 026107 (2009).

[9] B. Georgeot, O. Giraud, and D. L. Shepelyansky, Phys. Rev. E 81, 056109 (2010).

[10] K. M. Frahm, B. Georgeot, and D. L. Shepelyansky, J. Phys, A: Math. Theor. 44, 465101 (2011).

[11] L. Ermann, A. D. Chepelianskii, and D. L. Shepelyansky, Eur. Phys. J. B 79, 115 (2011).

[12] K. M. Frahm and D. L. Shepelyansky, Eur. Phys. J. B 85, 355 (2012).

[13] L. Ermann, K. M. Frahm, and D. L. Shepelyansky, Eur. Phys. J. B 86, 193 (2013).

[14] Y.-H. Eom, K. M. Frahm, A. Benczur, and D. L. Shepelyansky, Eur. Phys. J. B 86, 492 (2013).

[15] R. Albert and A.-L. Barabási, Phys. Rev. Lett. 85, 5234 (2000).

[16] Web page of Physical Review: http://publish.aps.org/.

[17] G. W. Stewart, Matrix Algorithms, Eigensystems (SIAM, Washington, DC, 2001), Vol. II.

[18] K. M. Frahm and D. L. Shepelyansky, Eur. Phys. J. B 76, 57 (2010).

[19] K. M. Frahm, A. D. Chepelianskii, and D. L. Shepelyansky, J. Phys. A: Math. Theor. 45, 405101 (2012).

[20] S. Redner, Phys. Today 58(6), 49 (2005).

[21] P. Chen, H. Xie, S. Maslov, and S. Redner, J. Infometrics 1, 8 (2007).

[22] F. Radicchi, S. Fortunato, B. Markines, and A. Vespignani, Phys. Rev. E 80, 056103 (2009).
[23] Y.-H. Eom and S. Fortunato, PLoS ONE 6, e24926 (2011).

[24] J. D. West, T. C. Bergstrom, and C. T. Bergstrom, Coll. Res. Libr. 71, 236 (2010); http://www.eigenfactor.org/.

[25] A. D. Chepelianskii, arXiv:1003.5455 [cs.Se] (2010).

[26] A. O. Zhirov, O. V. Zhirov, and D. L. Shepelyansky, Eur. Phys. J. B 77, 523 (2010)

[27] L. Ermann, A. D. Chepelianskii, and D. L. Shepelyansky, J. Phys. A: Math. Theor. 45, 275101 (2012).

[28] This number depends on the exact time ordering which is used and which is not unique because many papers are published at the same time and the order between them is not specified. We have chosen a time ordering where between these papers, degenerate in publication time, the initial node order of the raw data is kept.

[29] Note that some of the nonvanishing components of the iteration vector $S_{0}^{i} e$ may become very small, e.g., $\sim 10^{-100}$. In this context we count such components still as occupied despite their small size, and $N_{i}$ is the number of nodes which can be reached from some arbitrary other node after $i$ iterations with the matrix $S_{0}$.

[30] T. Granlund and the GMP development team, http://gmplib.org/.

[31] J. Sjöstrand, Duke Math. J. 60, 1 (1990).

[32] J. Sjöstrand and M. Zworski, Duke Math. J. 137, 381 (2007).

[33] S. Nonnenmacher and M. Zworski, Commun. Math. Phys. 269, 311 (2007).

[34] J. Bardeen, L. N. Cooper, and J. R. Schrieffer, Phys. Rev. 108, 1175 (1957)

[35] P. W. Anderson, Phys. Rev. 109, 1492 (1958).

[36] G. Benettin, L. Galgani, and J.-M. Strelcyn, Phys. Rev. A 14, 2338 (1976)

[37] D. J. Thouless, Phys. Rev. Lett. 39, 1167 (1977).

[38] E. Abrahams, P. W. Anderson, D. C. Licciardello, and T. V. Ramakrishnan, Phys. Rev. Lett. 42, 673 (1979).

[39] Y.-H. Eom and D. L. Sepelyansky, PLoS ONE 8, e74554 (2013).

[40] J. Ginibre, J. Math. Phys. Sci. 6, 440 (1965).

[41] H.-J. Sommers, A. Crisanti, H. Sompolinsky, and Y. Stein, Phys. Rev. Lett. 60, 1895 (1988); N. Lehmann and H.-J. Sommers, ibid. 67, 941 (1991).

[42] V. Kandiah and D. L. Shepelyansky, PLoS ONE 8, e61519 (2013).

[43] In Ref. [19] a set of vectors without this prefactor was used, but this provided a representation matrix which is numerically unstable for a direct diagonalization. The prefactor $c_{j-1}^{-1}$ ensures that the representation matrix is numerically (rather) stable, and of course both matrices are mathematically related by a similarity transformation and have identical eigenvalues.

[44] J. Stoer and R. Bulirsch, Introduction to Numerical Analysis (Springer, New York, 2002). 